\title{
Master Catalogue of Lunar and Mars Exploration Missions
}

\author{
Malaya Kumar Biswal $\mathbf{M}^{*}$ and Ramesh Naidu Annavarapu* \\ Department of Physics, School of Physical Chemical and Applied Sciences, \\ Pondicherry University, Kalapet, Puducherry, India - 605014
}

\begin{abstract}
This paper contains catalogues of Chronology of Lunar and Mars Exploration Missions with their respective probe parameters such as launch mass, probe mass, the period of mission duration and mission degradation, mission intervals between launches. The novel feature of this catalogues are precise and verified data gathered from reliable online resources and journal articles than other resources available. These catalogues are assorted to have clearance in mission attempts and trends since the last century towards the Moon and Mars. It also encloses random graphical images generated using the data mentioned in this catalogue. The catalogues also comprise gravity assist probes and test launches. Some of the unclear data were gathered from online resources and have uncertain in data validation. This catalogue is derived from our previous complied works described in the supplementary materials section.
\end{abstract}

\section{Nomenclature}

\begin{tabular}{|c|c|}
\hline AIMP & $=$ Anchored Interplanetary Monitoring Platform \\
\hline$A R C$ & $=$ Ames Research Center \\
\hline$B M D O$ & $=$ Ballistic Missile Defense Organization \\
\hline CAST & = China Academy of Space Technology \\
\hline$C C-L C$ & $=$ Cape Canaveral Launch Complex \\
\hline$C C-S L C$ & $=$ Cape Canaveral Space Launch Complex \\
\hline CNSA & $=$ China National Space Administration \\
\hline DSPSE & $=$ Deep Space Program Science Experiment \\
\hline$D S W L P$ & $=$ Discovering the Sky at Longest Wavelengths Pathfinder \\
\hline$E L A$ & $=$ Ensemble de Lancement Ariane \\
\hline$E L S$ & $=$ Ensemble de Lancement Soyuz \\
\hline ESA & $=$ European Space Agency \\
\hline ESPD & = Elapsed \\
\hline$G G S$ & $=$ Global Geospace Science \\
\hline$G R A I L$ & $=$ Gravity Recovery and Interior Laboratory \\
\hline GSFC & $=$ Goddard Space Flight Center \\
\hline GSLV & $=$ Geosynchronous Satellite Launch Vehicle \\
\hline GSMZ & $=$ Lavochkin Research and Production Association (NPO) \\
\hline$H I T$ & $=$ Harbin Institute of Technology \\
\hline$I A I$ & $=$ Israel Aerospace Industries \\
\hline$I C E$ & $=$ International Cometary Explorer \\
\hline ISAS & $=$ Institute of Space and Aeronautical Science \\
\hline ISEE & $=$ International Sun $=$ Earth Explorer \\
\hline ISRO & $=$ Indian Space Research Organisation \\
\hline$J A X A$ & = Japanese Aerospace Exploration Agency \\
\hline JHUAPL & $=$ The John Hopkins University Applied Physics Laboratory \\
\hline$J P L$ & $=$ Jet Propulsion Laboratory \\
\hline$\angle A D E E$ & $=$ Lunar Atmosphere and Dust Environment Explorer \\
\hline
\end{tabular}

\footnotetext{
* Graduate Researcher, Department of Physics, Pondicherry University, India; malaykumar1997@ gmail.com, mkumar97.res@pondiuni.edu.in, Member of Indian Science Congress Association, Student Member AIAA

‡ Associate Professor, Department of Physics, Pondicherry University, India; rameshnaidu.phy@ pondiuni.edu.in,arameshnaidu@gmail.com.
} 


\begin{tabular}{|c|c|}
\hline LaRC & $=$ Langley Research Center \\
\hline LCROSS & $=$ Lunar Crater Observation and Sensing Satellite \\
\hline$L M$ & $=$ Lunar Module \\
\hline LMSS & $=$ Lockheed Martin Space Systems \\
\hline$L O K$ & $=$ Lunniy Orbitalny Korabl \\
\hline$L R O$ & $=$ Lunar Reconnaissance Orbiter \\
\hline$L U X$ & $=$ Luxemburg \\
\hline$L V$ & $=$ Launch Vehicle \\
\hline$M A R S-L P$ & $=$ Mid=Atlantic Regional Spaceport Launch Pad \\
\hline MAVEN & $=$ Mars Atmosphere and Volatile Evolution \\
\hline$M C O$ & $=$ Mars Climate Orbiter \\
\hline$M E R$ & $=$ Mars Exploration Rover \\
\hline$M G S$ & $=$ Mars Global Surveyor \\
\hline MIP & $=$ Moon Impact Probe \\
\hline$M I T$ & $=$ Massachusetts Institute of Technology \\
\hline$M M M M$ & $=$ Manfred Memorial Moon Mission \\
\hline$M O M$ & $=$ Mars Orbiter Mission \\
\hline$M P L$ & $=$ Mars Polar Lander \\
\hline$M R O$ & $=$ Mars Reconnaissance Orbiter \\
\hline$M S$ & $=$ Mars Surveyor \\
\hline$M S L$ & $=$ Mars Science Laboratory \\
\hline$M V$ & $=$ Mars Venus \\
\hline$N A A$ & $=$ North American Aviation \\
\hline$N A R$ & $=$ North American Rockwell Grumman \\
\hline$N A S A$ & $=$ National Aeronautics and Space Administration \\
\hline NASA & $=$ National Aeronautics Space Administration \\
\hline$N P O$ & $=$ Lavochkin Research and Production Association \\
\hline$N R A O$ & $=$ National Radio Astronomy Observatory \\
\hline$O K B$ & $=$ Opytnoye Konstruktorskoye Buro (Experimental Design Bureau) \\
\hline PAS 22 & $=$ PanAm Sat \\
\hline$P F S$ & $=$ Particles $\&$ Fields Sub $=$ satellite \\
\hline PSLV & $=$ Polar Satellite Launch Vehicle \\
\hline$R A E$ & $=$ Radio Astronomy Explorer \\
\hline$R S A T$ & $=$ Radio Satellite \\
\hline SANSEI & $=$ Shanghai Aerospace System Engineering Institute \\
\hline SELENE & $=$ Selenological and Engineering Explorer \\
\hline SHAR & $=$ Sriharikota Launch Pad \\
\hline$S L C$ & $=$ Space Launch Complex \\
\hline$S L V$ & $=$ Space Launch Vehicle \\
\hline SMART & $=$ Small Missions for Advanced Research in Technology \\
\hline$S S C$ & $=$ Swedish Space Corporation \\
\hline STEREO & $=$ Solar Terrestrial Relations Observatory \\
\hline$S T L$ & $=$ Space Technology Laboratories \\
\hline STS & $=$ Space Transportation System \\
\hline$T A S$ & $=$ Thales Alena Space \\
\hline TESS & $=$ Transiting Exoplanet Survey Satellite \\
\hline$T s K B E M$ & $=$ S.P. Korolev Rocket and Space Corporation Energia \\
\hline$T S S$ & $=$ Toshiba Space Systems \\
\hline$T Y$ & = Tanegashima Yoshinobu 1 \\
\hline$U S$ & $=$ United States \\
\hline USC & $=$ Uchinoura Space Center \\
\hline USSR & $=$ Union of Soviet Socialist Republics (Russia) \\
\hline$V R A D$ & $=$ Very-Long-Baseline-Interferometer Radio Source \\
\hline WMAP & $=$ Wilkinson Microwave Anisotropy Probe \\
\hline$X L$ & $=$ Extra Large \\
\hline$X L S / X S L C$ & $=$ Xichang Satellite Launch Center \\
\hline
\end{tabular}


Table-1 Chronology of Lunar Exploration Missions

\begin{tabular}{|c|c|c|c|c|c|c|c|c|c|c|}
\hline S.No & Spacecraft & Distinct Name & Type & Manufacturer & Launch Date & $\begin{array}{c}\text { End of Mission } \\
\text { Decay Date }\end{array}$ & Launcher & Launcher Type & Launch Site & Country \\
\hline 01. & Pioneer 0 & Able-1 & Orbiter & STL & 17-Aug-1958 & 17-Aug-1958 & Thor & DM-18-Able-1 & CC-LC-17A & US \\
\hline 02. & Luna E-1 No.1 & & Impactor & OKB-1 & 23-Sep-1958 & 23-Sep-1958 & Luna 8K72 & S.No. B1-3 & Baikonur 1/5 & USSR \\
\hline 03. & Pioneer 1 & Able-2 & Orbiter & STL & 11-Oct-1958 & 13-Oct-1958 & Thor & DM-18-Able-1 & CC-LC-17A & US \\
\hline 04. & Luna E-1.No.2 & & Impactor & OKB-1 & 12-Oct-1958 & 12-Oct-1958 & Luna 8K72 & S.No. B1-4 & Baikonur 1/5 & USSR \\
\hline 05. & Pioneer 2 & Able-3 & Orbiter & STL & 08-Nov-1958 & 08-Nov-1958 & Thor & DM-18-Able-1 & CC-LC-17A & US \\
\hline 06. & Luna E-1.No.3 & & Impactor & OKB-1 & 04-Dec-1958 & 04-Dec-1958 & Luna 8K72 & S.No. B1-5 & Baikonur 1/5 & USSR \\
\hline 07. & Pioneer 3 & & Flyby & JPL & 06-Dec-1958 & 07-Dec-1958 & Juno & Version - II & CC-LC-05 & US \\
\hline 08. & Mechta /Luna 1 & Luna E-1.No.4 & Impactor & OKB-1 & 02-Jan-1959 & 05-Jan-1959 & Luna 8K72 & S.No. B1-6 & Baikonur 1/5 & USSR \\
\hline 09. & Pioneer 4 & & Flyby & JPL & 03-Mar-1959 & 06-Mar-1959 & Juno & Version - II & CC-LC-05 & US \\
\hline 10. & Luna E-1A.No.1 & Luna E-1A.No.5 & Impactor & OKB-1 & 18-Jun-1959 & 18-Jun-1959 & Luna 8K72 & S.No. L1-7 & Baikonur 1/5 & USSR \\
\hline 11. & Luna 2 & Luna E-1A.No.2 & Impactor & OKB-1 & 12-Sep-1959 & 14-Sep-1959 & Luna 8K72 & S.No. L1-7B & Baikonur 1/5 & USSR \\
\hline 12. & Pioneer P-1 & & Orbiter & STL & 24-Sep-1959 & 26-Sep-1959 & Atlas-C & Able & CC-LC-12 & US \\
\hline 13. & Luna 3 & Luna E-2A.No.1 & Flyby & OKB-1 & 04-Oct-1959 & $22-$-Oct-1959 & Luna 8K72 & S.No. L1-8 & Baikonur 1/5 & USSR \\
\hline 14. & Pioneer P-3 & Able-IVB & Orbiter & STL & 26-Nov-1959 & 26-Nov-1959 & Atlas-D & Able & CC-LC-14 & US \\
\hline 15. & Luna 1960A & Luna E-3.No.1 & Circumlunar & OKB-1 & 15 -Apr-1960 & 15 -Apr-1960 & Luna 8K72 & S.No.L1-9 & Baikonur 1/5 & USSR \\
\hline 16. & Luna 1960B & Luna E-3.No.2 & Circumlunar & OKB-1 & 19-Apr-1960 & 19-Apr-1960 & Luna 8K72 & S.No.L1-9A & Baikonur 1/5 & USSR \\
\hline 17. & Pioneer P-30 & Able-VA & Orbiter & STL & 25-Sep-1960 & 25-Sep-1960 & Atlas-D & Able & CC-LC-12 & US \\
\hline 18. & Pioneer P-31 & Able-VB & Orbiter & STL & 15-Dec-1960 & 15-Dec-1960 & Atlas-D & Able & CC-LC-12 & US \\
\hline 19. & Ranger 1 & $\mathrm{P}-32$ & Deep Space & JPL & 23-Aug-1961 & 30-Aug-1961 & Atlas & Agena-B-1 & CC-LC-12 & US \\
\hline 20. & Ranger 2 & $\mathrm{P}-33$ & Deep Space & JPL & 18-Nov-1961 & 19-Nov-1961 & Atlas & Agena-B-2 & CC-LC-12 & US \\
\hline 21. & Ranger 3 & P-34 & Impactor & JPL & 26-Jan-1962 & 02-Feb-1962 & Atlas LV-3 & Agena-B & CC-LC-12 & US \\
\hline 22. & Ranger 4 & P-35 & Impactor & JPL & $23-A p r-1962$ & 26-Apr-1962 & Atlas LV-3 & Agena-B & CC-LC-12 & US \\
\hline 23. & Ranger 5 & P-36 & Impactor & JPL & 18-Oct-1962 & 21-Oct-1962 & Atlas LV-3 & Agena-B215D-AA7 & CC-LC-12 & US \\
\hline 24. & Sputnik 25 & Luna E-6.No.2 & Lander & OKB-1 & 04-Jan-1963 & 11-Jan-1963 & Molniya-L & 8K78-S.No.T103-09 & Baikonur 1/5 & USSR \\
\hline 25. & Luna E-6.No.3 & Luna E-6.No.3 & Lander & OKB-1 & 03-Feb-1963 & 03-Feb-1963 & Molniya-L & 8K78-S.No.G103-10 & Baikonur 1/5 & \begin{tabular}{|l|} 
USSR \\
\end{tabular} \\
\hline 26. & Luna 4 & Luna E-6.No.4 & Lander & OKB-1 & 02-Apr-1963 & 15-Apr-1963 & Molniya-L & 8K78/E6/G103-11 & Baikonur 1/5 & USSR \\
\hline 27. & Kosmos 21 & Cosmos 21 & L1 Test & OKB-1 & 11-Nov-1963 & 14-Nov-1963 & Molniya-L & 8K78M.S.No.G103-18 & Baikonur 1/5 & USSR \\
\hline 28. & Ranger 6 & $\mathrm{P}-53$ & Impactor & JPL & 30-Jan-1964 & 02-Feb-1964 & Atlas LV-3 & Agena-B/199D/AA8 & CC-LC-12 & US \\
\hline 29. & Luna 1964A & Luna E-6.No.6 & Lander & OKB-1 & 21-Mar-1964 & 21-Mar-1964 & Molniya-M & 8K78-M/T15000-20 & Baikonur $1 / 5$ & USSR \\
\hline 30. & Luna E-6.No.5 & Luna E-6.No.5 & Lander & OKB-1 & 20-Apr-1964 & 20-Apr-1964 & Molniya-M & $8 \mathrm{~K} 78 \mathrm{~L} / \mathrm{R} 103-25$ & Baikonur 1/5 & USSR \\
\hline 31. & Ranger 7 & P-54 & Impactor & JPL & 28-Jul-1964 & 31-Jul-1964 & Atlas LV-3 & Agena-B-9 & CC-LC-12 & US \\
\hline 32. & Ranger 8 & Ranger-C & Impactor & JPL & 17-Feb-1965 & 20-Feb-1965 & Atlas LV-3 & Agena-B-13 & CC-LC-12 & US \\
\hline 33. & Kosmos 60 & Luna E-6.No.9 & Lander & OKB-1 & 12-Mar-1965 & 17-Mar-1965 & Molniya-M & $8 \mathrm{~K} 78 \mathrm{~L} / \mathrm{R} 103-26$ & Baikonur 1/5 & USSR \\
\hline 34. & Ranger 9 & Ranger-D & Impactor & JPL & 21-Mar-1965 & 24-Mar-1965 & Atlas LV-3 & Agena-B-14 & CC-LC-12 & US \\
\hline 35. & Luna $1965 \mathrm{~A}$ & Luna E-6.No.8 & Lander & OKB-1 & 10-Apr-1965 & 10-Apr-1965 & Molniya-M & 8K78-M/R103-26 & Baikonur 1/5 & USSR \\
\hline 36. & Luna 5 & Luna E-6.No.10 & Lander & OKB-1 & 09-May-1965 & 12-May-1965 & Molniya-M & 8K78-M/U103-30 & Baikonur 1/5 & USSR \\
\hline 37. & Luna 6 & Luna E-6.No.7 & Lander & OKB-1 & 08-Jun-1965 & 18-Jun-1965 & Molniya-M & 8K78-M/U103-31 & Baikonur 1/5 & USSR \\
\hline 38. & Zond 3 & 3MV-4.No.3 & Flyby & OKB-1 & 18-Jul-1965 & 03-Mar-1966 & Molniya-M & $8 \mathrm{~K} 78-\mathrm{M}$ & Baikonur 1/5 & USSR \\
\hline 39. & Luna 7 & Luna E-6.No.11 & Lander & OKB-1 & 04-Oct-1965 & 07-Oct-1965 & Molniya-M & 8K78-M/U103-27 & Baikonur 1/5 & USSR \\
\hline 40. & Luna 8 & Luna E-6.No.12 & Lander & OKB-1 & 03-Dec-1965 & 06-Dec-1965 & Molniya-M & $8 \mathrm{~K} 78-\mathrm{M} / \mathrm{U} 103-28$ & Baikonur 31/6 & USSR \\
\hline 41. & Luna 9 & Luna E-6.No.13 & Lander & GSMZ-Lavochkin & 31-Jan-1966 & 06-Feb-1966 & Molniya-M & $8 \mathrm{~K} 78 \mathrm{M} / \mathrm{U} 103-32$ & Baikonur 31/5 & USSR \\
\hline 42. & Kosmos 111 & Luna E-6S.No.204 & Orbiter & OKB-1 & 01-Mar-1966 & 03-Mar-1966 & Molniya-M & $8 \mathrm{~K} 78-\mathrm{M} / \mathrm{N} 103-41$ & Baikonur 31/6 & USSR \\
\hline 43. & Luna 10 & Luna E-6S.No.206 & Orbiter & GSMZ-Lavochkin & 31-Mar-1966 & 30-May-1966 & Molniya-M & $8 \mathrm{~K} 78 \mathrm{M} / \mathrm{N} 103-42$ & Baikonur 31/5 & USSR \\
\hline 44. & Luna 1966A & & Orbiter & OKB-1 & 30-Apr-1966 & 30-Apr-1966 & Proton K/D & UR-500K & Baikonur 1/5 & USSR \\
\hline
\end{tabular}




\begin{tabular}{|c|c|c|c|c|c|c|c|c|c|c|}
\hline S.No & Spacecraft & Distinct Name & Type & Manufacturer & Launch Date & $\begin{array}{l}\text { End of Mission } \\
\text { Decay Date }\end{array}$ & Launcher & Launcher Type & Launch Site & Country \\
\hline 45. & Surveyor 1 & Surveyor-A & Lander & NASA/JPL & 30-May-1966 & 07-Jan-1967 & Atlas LV-3C & Centaur-D-10 & CC-LC-36A & US \\
\hline 46. & Explorer 33 & AIMP-D & Orbiter & NASA/GSFC & 01-Jul-1966 & 21-Sep-1976 & Delta & E1 & CC-LC-17A & US \\
\hline 47. & Lunar Orbiter 1 & Luna Orbiter-A & Orbiter & NASA/LaRC & 10-Aug-1966 & 29-Oct-1966 & Atlas SLV-3 & Agena-D & CC-LC-13 & US \\
\hline 48. & Luna 11 & E-6LF.No.101 & Orbiter & GSMZ Lavochkin & 21-Aug-1966 & 01-Oct-1966 & Molniya-M & 8K78-M & Baikonur 31/6 & USSR \\
\hline 49. & Surveyor 2 & Surveyor-B & Lander & NASA/JPL & 20-Sep-1966 & 23-Sep-1966 & Atlas LV-3C & Centaur-D & CC-LC-36A & US \\
\hline 50. & Luna 12 & E-6LF.No.102 & Orbiter & GSMZ Lavochkin & 22-Oct-1966 & 19-Jan-1967 & Molniya-M & $8 \mathrm{~K} 78 \mathrm{M} / \mathrm{N} 103-44$ & Baikonur 31/5 & USSR \\
\hline 51. & Lunar Orbiter 2 & Luna Orbiter-B & Orbiter & NASA/LaRC & 06-Nov-1966 & 11-Oct-1967 & Atlas LV-3 & Agena-D-18 & CC-LC-13 & US \\
\hline 52. & Luna 13 & E-6M.No.205 & Lander & GSMZ Lavochkin & 21-Dec-1966 & 28-Dec-1966 & Molniya-M & 9K78M/N103-45 & Baikonur 1/5 & USSR \\
\hline 53. & Apollo 1 & & Crewed Orbiter & NAA & 27-Jan-1967 & 27-Jan-1967 & Saturn V & AS-204 & CK-LC-34A & US \\
\hline 54. & Lunar Orbiter 3 & Luna Orbiter-C & Orbiter & NASA/LaRC & 05-Feb-1967 & 09-Oct-1967 & Atlas LV-3 & Agena-D-20 & CC-LC-13 & US \\
\hline 55. & Kosmos 146 & 7K-L1.No.2P & L1 Test & OKB-1 & 10-Mar-1967 & 22-Mar-1967 & Proton K/D & USR500K-N10722701 & Baikonur 1/5 & USSR \\
\hline 56. & Kosmos 154 & 7K-L1.No.3P & L1 Test & OKB-1 & 08-Apr-1967 & 10-Apr-1967 & Proton K/D & UR-500K & Baikonur 81/36 & USSR \\
\hline 57. & Surveyor 3 & Surveyor-C & Lander & NASA/JPL & 17-Apr-1967 & 03-Мay-1967 & Atlas LV-3C & Centaur-D-12 & CC-LC-36B & US \\
\hline 58. & Lunar Orbiter 4 & Luna Orbiter-D & Orbiter & NASA/LaRC & 04-May-1967 & 06-Oct-1967 & Atlas LV-3 & Agena-D-22 & CC-LC-13 & US \\
\hline 59. & Kosmos 159 & E6LS.No.111 & Orbiter & GSMZ Lavochkin & 17-May-1967 & 11-Nov-1967 & Molniya-M & $8 \mathrm{~K} 78 \mathrm{M} / \mathrm{A} 716-56$ & Baikonur 1/5 & USSR \\
\hline 60. & Surveyor 4 & Surveyor-D & Lander & NASA/JPL & 14-Jul-1967 & 17-Jul-1967 & Atlas LV-3C & Centaur-D & CC-LC-36A & US \\
\hline 61. & Explorer 35 & AIMP-E & Orbiter & NASA/GSFC & 19-Jul-1967 & 24-Jun-1973 & Thor & Delta-E1/50 & CC-LC-17B & US \\
\hline 62. & Lunar Orbiter 5 & Luna Orbiter-E & Orbiter & NASA/LaRC & 01-Aug-1967 & 31-Jan-1968 & Atlas LV-3 & Agena-D-24 & CC-LC-13 & US \\
\hline 63. & Surveyor 5 & Surveyor-E & Lander & NASA/JPL & 08-Sep-1967 & 17-Dec-1967 & Atlas-LV-3C & Centaur-D-13 & CC-LC-36B & US \\
\hline 64. & Zond 1967A & Soyuz 7K-L1.No.4L & Flyby & TsKBEM & $27-$ Sep-1967 & 27-Sep-1967 & Proton K/D & S.No.229-01 & Baikonur $81 / 23$ & USSR \\
\hline 65. & Surveyor 6 & Surveyor-F & Lander & NASA/JPL & 07-Nov-1967 & 14-Dec-1967 & Atlas-LV-3C & Centaur-D-14 & CC-LC-36B & US \\
\hline 66. & Zond 1967B & Soyuz 7L-L1.No.5L & Flyby & TsKBEM & 22-Nov-1967 & 22-Nov-1967 & Proton K/D & S.No.230-01 & Baikonur 81/24 & USSR \\
\hline 67. & Surveyor 7 & Surveyor-G & Lander & NASA/JPL & 07-Jan-1968 & 21-Feb-1968 & Atlas LV-3C & Centaur-D-15 & CC-LC-36A & US \\
\hline 68 & Luna 1968A & Luna E-6LS.No.112 & Orbiter & GSMZ Lavochkin & 07-Feb-1968 & 07-Feb-1968 & Molniya-M & $\begin{array}{l}\text { 8K78M.S.No.YG716- } \\
57\end{array}$ & Baikonur 1/5 & USSR \\
\hline 69. & Zond 4 & 7K-L1.No.6L & Deep Space & TsKBEM & 02-Mar-1968 & 07-Mar-1968 & Proton K/D & UR-500K & Baikonur $81 / 6$ & USSR \\
\hline 70. & Luna 14 & E-6LS.No.113 & Orbiter & GSMZ Lavochkin & 07-Apr-1968 & 24-Jun-1968 & Molniya-M & 8K78/A716-58 & Baikonur 1/5 & USSR \\
\hline 71. & Zond 1968A & Soyuz 7K-L1.7L & Flyby & TsKBEM & 22-Apr-1968 & 22-Apr-1968 & Soyuz & 7K-L1.S.No.7 & Baikonur 1/5 & USSR \\
\hline 72. & Zond 5 & 7K-L1.No.9L & Circumlunar & TsKBEM & 14-Sep-1968 & 21-Sep-1968 & Proton K/D & UR-500K/234-01 & Baikonur $81 / 5$ & USSR \\
\hline 73. & Apollo 7 & & Crewed Orbiter & NAR & $11-$ Oct-1968 & $22-$ Oct-1968 & Saturn IB & SA-205 & CK-LC-34 & US \\
\hline 74. & Zond 6 & 7K-L1.No.12L & Flyby & TsKBEM & 10-Nov-1968 & 17-Nov-1968 & Proton-K/D & $11 \mathrm{~S} 824$ & Baikonur 81/26 & USSR \\
\hline 75. & Apollo 8 & & Crewed Orbiter & NAR & 21-Dec-1968 & 27-Dec-1968 & Saturn V & AS-503 & CC-LC-39A & US \\
\hline 76. & Zond 1969A & Soyuz 7K-L1.No.13L & Flyby & TsKBEM & 20-Jan-1969 & 20-Jan-1969 & Soyuz & 7K-L1.S.No.13 & Baikonur 81/24 & USSR \\
\hline 77. & Luna 1969A & Luna E-8.No.201 & Lander/Rover & GSMZ Lavochkin & 19-Feb-1969 & 19-Feb-1969 & Proton K/D & S.No.239-01 & Baikonur 81/24 & USSR \\
\hline 78. & Zond L1S-1 & Soyuz 7K-L1S.No.3S & Orbiter & TsKBEM & 21-Feb-1969 & 21-Feb-1969 & N1 & SL-15 & Baikonur 110/38 & USSR \\
\hline 79. & Apollo 9 & LM-Spider & Crewed Orbiter & NAR / Grumman & 03-Mar-1969 & 13-Mar-1969 & Saturn V & $\mathrm{AS}=504$ & CK-LC-39A & US \\
\hline 80. & Luna 1969B & & Sample Return & GSMZ Lavochkin & 15-Apr-1969 & 15-Apr-1969 & Proton K/D & UR-500K & Baikonur 81/24 & USSR \\
\hline 81. & Apollo 10 & LM-Snoopy & Crewed Orbiter & NAR / Grumman & 18-Мay-1969 & 26-Мay-1969 & Saturn V & AS-505 & CC-LC-39B & US \\
\hline 82. & Luna 1969C & Luna E-8-5.No.402 & Sample Return & GSMZ Lavochkin & 14-Jun-1969 & 14-Jun-1969 & Proton-K/D & UR-500K & Baikonur 81/24 & USSR \\
\hline 83. & Zond L1S-2 & Soyuz 7L-L1S.No.5L & Orbiter & TsKBEM & 03-Jul-1969 & 03-Jul-1969 & N1 & SL-15 & Baikonur 110/38 & USSR \\
\hline 84. & Luna 15 & E-8-5.No.401 & Sample Return & GSMZ Lavochkin & 13-Jul-1969 & 21-Jul-1969 & Proton K/D & UR-500K/242-01 & Baikonur 81/24 & USSR \\
\hline 85. & Apollo 11 & LM-Eagle & Crewed Orbiter & NAR / Grumman & 16-Jul-1969 & 24-Jul-1969 & Saturn V & $\mathrm{AS}=506$ & CC-LC-39A & US \\
\hline 86. & Zond 7 & 7K-L1.No.11L & Flyby & TsKBEM & 07-Aug-1969 & 14-Aug-1969 & Proton K/D & UR-500K/243-01 & Baikonur $81 / 5$ & USSR \\
\hline 87. & Kosmos 300 & E-8-5.No.403 & Sample Return & GSMZ Lavochkin & 23-Sep-1969 & 27-Sep-1969 & Proton K/D & UR-500K/244-01 & Baikonur $81 / 5$ & USSR \\
\hline 88. & Kosmos 305 & E-8-5.No.404 & Sample Return & GSMZ Lavochkin & 22-Oct-1969 & 24-Oct-1969 & Proton K/D & UR-500K/241-01 & Baikonur $81 / 5$ & USSR \\
\hline 89. & Apollo 12 & LM-Intrepid & Crewed Orbiter & NAR / Grumman & 14-Nov-1969 & 24-Nov-1969 & Saturn V & AS-507 & CC-LC-39A & US \\
\hline
\end{tabular}




\begin{tabular}{|c|c|c|c|c|c|c|c|c|c|c|}
\hline S.No & Spacecraft & Distinct Name & Type & Manufacturer & Launch Date & $\begin{array}{c}\text { End of Mission } \\
\text { Decay Date }\end{array}$ & Launcher & Launcher Type & Launch Site & Country \\
\hline 90. & Luna 1970A & Luna E-8-5.No.405 & Sample Return & GSMZ Lavochkin & 06-Feb-1970 & 06-Feb-1970 & Proton K/D & S.No.247-01 & Baikonur 81/23 & USSR \\
\hline 91. & Luna 1970B & & Orbiter & GSMZ Lavochkin & $19-F e b-1970$ & 19-Feb-1970 & Proton K/D & S.No.12/D-1e & Baikonur $81 / 23$ & USSR \\
\hline 92. & Apollo 13 & LM-Aquarius & Crewed Orbiter & NAR / Grumman & 11-Apr-1970 & 17-Apr-1970 & Saturn V & AS-508 & CC-LC-39A & US \\
\hline 93. & Luna 16 & E-8-5.No.406 & Sample Return & GSMZ Lavochkin & 12-Sep-1970 & 24-Sep-1970 & Proton K/D & SL-12/D-1.E & Baikonur 81/24 & USSR \\
\hline 94. & Zond 8 & 7K-L1.No.14L & Flyby & TsKBEM & $20-$ Oct-1970 & $27-$ Oct-1970 & Proton K/D & UR-500K/250-01 & Baikonur $81 / 5$ & USSR \\
\hline 95. & Luna 17 & E-8.No.203 & Lander/Rover & GSMZ Lavochkin & 10-Nov-1970 & 17-Nov-1971 & Proton K/D & UR-500K/251-01 & Baikonur 81/5 & USSR \\
\hline 96. & Apollo 14 & LM-Antares & Crewed Orbiter & NAR / Grumman & 31-Jan-1971 & 09-Feb-1971 & Saturn V & AS-509 & CC-LC-39A & US \\
\hline 97. & Soyuz 7K-LOK & 7K-L1E.No.1 & Circumlunar & TsKBEM & 27-Jun-1971 & 27-Jun-1971 & N1 & $\mathrm{N} 1$ & Baikonur 110/37 & USSR \\
\hline 98. & Apollo 15 & LM-Falcon & Crewed Orbiter & NAR / Grumman & 26-Jul-1971 & 23-Aug-1973 & Saturn V & AS-510 & CC-LC-39A & US \\
\hline 99. & PFS-1 & & Orbiter & NASA & 26-Jul-1971 & 23-Aug -1973 & Saturn V & AS-510 & CC-LC-39A & US \\
\hline 100. & Luna 18 & E-8-5.No.407 & Sample Return & GSMZ Lavochkin & 02-Sep-1971 & 11-Sep-1971 & Proton K/D & 8K82K /S.No.256-01 & Baikonur 81/24 & USSR \\
\hline 101. & Luna 19 & E-8LS.No.202 & Orbiter & GSMZ Lavochkin & 28-Sep-1971 & $20-$ Oct-1972 & Proton K/D & UR-500K/257-01 & Baikonur $81 / 5$ & USSR \\
\hline 102. & Luna 20 & E-8-5.No.408 & Sample Return & GSMZ Lavochkin & 14-Feb-1972 & $25-F e b-1972$ & Proton K/D & UR-500K/258-01 & Baikonur $81 / 5$ & USSR \\
\hline 103. & Apollo 16 & LM-Orion & Crewed Orbiter & NAR / Grumman & 16-Apr-1972 & 27-Apr-1972 & Saturn V & AS-511 & CC-LC-39A & US \\
\hline 104. & PFS-2 & & Orbiter & NASA & 16-Apr-1972 & 29-May-1972 & Saturn V & AS-511 & CC-LC-39A & US \\
\hline 105. & Soyuz 7K-LOK & Soyuz 7K-LOK.No.1 & Orbiter & TsKBEM & 03-Jul-1972 & 03-Jul-1972 & NI & NI & Baikonur 110/37 & USSR \\
\hline 106. & Soyuz 7K-LOK & 7K-LOK.No.6A & Circumlunar & TsKBEM & 23-Nov-1972 & 23-Nov-1972 & N1 & N1 & Baikonur 110/37 & USSR \\
\hline 107. & Apollo 17 & LM-Challenger & Crewed Orbiter & NAR / Grumman & 07-Dec-1972 & 19-Dec-1972 & Saturn V & AS-512 & CC-LC-39A & US \\
\hline 108. & Luna 21 & E-8.No.204 & Lander/Rover & GSMZ Lavochkin & 08-Jan-1973 & 15-Jan-1973 & Proton K/D & UR-500K/259-01 & Baikonur $81 / 5$ & USSR \\
\hline 109. & Explorer 49 & RAE-B & Orbiter & NASA/GSFC & 10-Jun-1973 & 30-Aug-1977 & Delta & $1913-95$ & CC-LC-17B & US \\
\hline 110. & Mariner 10 & & Flyby & NASA/JPL & 03-Nov-1973 & 24-Mar-1975 & Atlas-SLV-3 & Centaur-D-34 & CC-LC-36B & US \\
\hline 111. & Luna22 & E-8LS.No.206 & Orbiter & GSMZ Lavochkin & 29-May-1974 & 10-Nov-1975 & Proton K/D & UR-500K/282-02 & Baikonur 81/5 & USSR \\
\hline 112. & Luna 23 & E-8-5M.No.410 & Sample Return & GSMZ Lavochkin & 28 -Oct-1974 & 09-Nov-1974 & Proton K/D & 8K82K/S.No.285-01 & Baikonur 81/24 & USSR \\
\hline 113. & Luna 1975A & Luna E-8-5.No.412 & Sample Return & NPO Lavochkin & 16-Oct-1975 & 23-Oct-1975 & Proton K/D & SL-12/D-1.E & Baikonur 81/23 & USSR \\
\hline 114. & Luna 24 & E-8-5M.No.413 & Sample Return & NPO Lavochkin & 09-Aug-1976 & 22-Aug-1976 & Proton K/D & UR-500K/288-02 & Baikonur $81 / 5$ & USSR \\
\hline 115. & ISEE-3 & Explorer 59/ICE & Gravity Assist & NASA/GSFC & 12-Aug-1978 & 05-May-1997 & Delta & $2914-144$ & CC-LC-41 & US \\
\hline 116. & Galileo & & Flyby & NASA/JPL & 18 -Oct-1989 & 21-Sep-2003 & STS-34R & IUS & CC-LC-39B & US \\
\hline 117. & Hiten & MUSES-A & Flyby/Orbiter & ISAS & 24-Jan-1990 & 10-Apr-1993 & MU-3S11 & S.No.5-3S11 & Launch Complex M1 & JAPAN \\
\hline 118. & Hagoromo & & Orbiter & ISAS & 24-Jan-1990 & 10-Apr-1993 & $\mathrm{Mu}-3511$ & S.No.5-3S11 & Launch Complex M1 & JAPAN \\
\hline 119. & Geotail & & Gravity Assist & ISAS & 24-Jul-1992 & EPSD:20 Years & Delta-II & 6925 & CC-LC-17A & US \\
\hline 120. & Clementine & DSPSE & Orbiter & NASA/BMDO & 25-Jan-1994 & 10-May-1995 & Titan-III & S.No.4 & Vandenberg SLC-4W & US \\
\hline 121. & WIND & GGS & Gravity Assist & NASA/GSFC & 01-Nov-1994 & $\begin{array}{l}\text { EPSD } \\
: 25 Y 4 M 13 \mathrm{D}\end{array}$ & Delta & $7925-10 / 227$ & CC-SLC-17B & US \\
\hline 122. & Cassini & Huygens & Flyby & NASA/JPL & 15-Oct-1997 & 15-Sep-2017 & Titan 401B & Centaur-D-21 & CC-LC-40 & US \\
\hline 123. & HGS-1 & Asiasat-3/PAS-22 & Gravity Assist & HUGHES & 24-Dec-1997 & 00-Jul-2002 & Proton-K & DM-3 & Baikonur & USSR \\
\hline 124. & Lunar Prospector & Discovery 3 & Orbiter & NASA/ARC & 07-Jan-1998 & 31-Jul-1999 & Athena-2 & LM-004 & Vandenberg SLC-46 & US \\
\hline 125. & Nozomi & Planet-B & Gravity Assist & ISAS & 03-Jul-1998 & 09-Dec-2003 & $\mathrm{M}-\mathrm{V}$ & $\mathrm{M}-\mathrm{V}$ & Launch Complex M5 & JAPAN \\
\hline 126. & WMAP & & Gravity Assist & NASA/NRAO & 30-Jun-2001 & $20-$ Oct-2010 & Delta-II & $7425-10$ & CC-SLC-17 & US \\
\hline 127. & SMART-1 & & Orbiter & SSC/ESA & 27-Sep-2003 & 03-Sep-2006 & Ariane 5G & $5 \mathrm{G}$ & Kourou ELS-3 & EUROPE \\
\hline 128. & STEREO-A & & Gravity Assist & JHUAPL/NASA & $25-$ Oct-2006 & Operational & Delta-II & $7925-10 \mathrm{~L}$ & CC-SLC-17B & US \\
\hline 129. & STEREO-B & & Gravity Assist & JHUAPL/NASA & 25-Oct-2006 & 23-Sep-2016 & Delta-II & $7925-10 \mathrm{~L}$ & CC-SLC-17B & US \\
\hline 130. & ARTEMIS-P1 & & Orbiter & Swales Aerospace & 17-Feb-2007 & Operational & Delta-II & $7925-10 \mathrm{C}$ & CC-SLC-17B & US \\
\hline 131. & ARTEMIS-P2 & & Orbiter & Swales Aerospace & 17-Feb-2007 & Operational & Delta-II & $1925-10 \mathrm{C}$ & CC-SLC-17B & US \\
\hline 132. & SELENE & Kaguya & Orbiter & NEC - TSS & 14-Sep-2007 & 10-Jun-2009 & H-IIA & $2022-\mathrm{F} 13$ & Tanegashima TY-1 & JAPAN \\
\hline 133. & Okina & RSAT & Orbiter & NEC - TSS & 14-Sep-2007 & 12-Feb-2009 & H-IIA & $2022-\mathrm{F} 13$ & Tanegashima TY-1 & JAPAN \\
\hline 134. & Ouna & VRAD & Orbiter & NEC - TSS & 14-Sep-2007 & 29-Jun-2009 & H-IIA & $2022-\mathrm{F} 13$ & TY-1 & JAPAN \\
\hline
\end{tabular}




\begin{tabular}{|c|c|c|c|c|c|c|c|c|c|c|}
\hline S.No & Spacecraft & Distinct Name & Type & Manufacturer & Launch Date & $\begin{array}{c}\text { End of Mission } \\
\text { Decay Date }\end{array}$ & Launcher & Launcher Type & Launch Site & Country \\
\hline 135. & Chang'e 1 & & Orbiter & CNSA & 24-Oct-2007 & 01-Mar-2009 & Long March & $3 \mathrm{~A}$ & XLC-3 & CHINA \\
\hline 136. & Chandrayaan 1 & & Orbiter & ISRO & 21-Oct-2008 & 28-Aug-2009 & PSLV-XL & $\mathrm{C} 11$ & SHAR & INDIA \\
\hline 137. & MIP & Moon Impact Probe & Impactor & ISRO & 21-Oct-2008 & 14-Nov-2008 & PSLV-XL & $\mathrm{C} 11$ & SHAR & INDIA \\
\hline 138 & LRO & & Orbiter & NASA/GSFC & 18-Jun-2009 & Operational & Atlas-V & 401 & CC-SLC-41 & US \\
\hline 139. & LCROSS & & Impactor & Northrop Grumman & 18-Jun-2009 & 09-Oct-2009 & Atlas-V & 401 & CC-SLC-41 & US \\
\hline 140. & Chang'e 2 & & Orbiter & CNSA & $01-$ Oct-2010 & $\begin{array}{l}\text { ESPD: 9Y 5M } \\
\text { 17D }\end{array}$ & Long March & $3 \mathrm{C}$ & XLC-3 & CHINA \\
\hline 141. & Ebb & GRAIL-A & Orbiter & MIT/LMSS & 10-Sep-2011 & 17-Dec-2012 & Delta-II & 7920H-10-D356 & CC-SLC-17B & US \\
\hline 142. & Flow & GRAIL-B & Orbiter & MIT/LMSS & 10-Sep-2011 & 17-Dec-2012 & Delta-II & $7920 \mathrm{H}-10 \mathrm{D}-356$ & CC-SLC-17B & US \\
\hline 143. & LADEE & & Orbiter & ARC & 07-Sep-2013 & 18-Apr-2014 & Minotaur V & Flight-1 & MARS-LP-0B & US \\
\hline 144. & Chang'e 3 & & Lander & SASEI & 01-Dec-2013 & Operational & Long March & $3 B-Y-23$ & XLC-2 & CHINA \\
\hline 145. & Yutu & & \begin{tabular}{|l|} 
Rover \\
\end{tabular} & \begin{tabular}{|l|} 
SASEI \\
SASEI
\end{tabular} & $01-$ Dec-2013 & 00-Mar-2015 & Long March & $3 B-Y-23$ & XLC-2 & CHINA \\
\hline 146. & Chang'e 5-T1 & & \begin{tabular}{|l|} 
Flyby \\
\end{tabular} & \begin{tabular}{|l} 
CAST \\
CANI
\end{tabular} & $23-$ Oct-2014 & $31-$ Oct-2014 & Long March & $3 \mathrm{C}-\mathrm{G}-2$ & XLC-2 & CHINA \\
\hline 147. & MMMM & 4M Mission & \begin{tabular}{|l} 
Flyby \\
\end{tabular} & LUX Space & $23-$ Oct-2014 & $11-$ Nov-2014 & Long March & $3 \mathrm{C}-\mathrm{G}-2$ & XLC-2 & CHINA \\
\hline 148. & TESS & & Gravity Assist & Orbital ATK & 18-Apr-2018 & ESPD:1Y 11M & Falcon 9 & Block-4 & CC-SLC-40 & US \\
\hline 149. & Queqiao & Chang'e 4 Relay & Gravity Assist & CNSA & 20-May-2018 & Operational & Long March & IV (4) & XLC-3 & CHINA \\
\hline 150. & Longjiang 1 & DSWLP-A1/LO-93 & Orbiter & CNSA/HIT & 20-May-2018 & 21-May-2018 & Long March & IV (4) & XLC-3 & CHINA \\
\hline 151. & Longjiang 2 & DSWLP-A2/LO-94 & Orbiter & CNSA & 20-May-2018 & 31-Jul-2019 & Long March & IV (4) & XLC-3 & CHINA \\
\hline 152. & Chang'e 4 & & Lander/Rover & CNSA & 07-Dec-2018 & ESPD: 440D & Long March & $3 \mathrm{~B}$ & XLC-3 & CHINA \\
\hline 153. & Beresheet & & Lander & IAI & 22-Feb-2019 & 04-Apr-2019 & Falcon & Falcon-9-B5 & CC-SLC-40 & ISRAEL \\
\hline 154. & Chandrayaan 2 & & Orbiter & ISRO & 22-Jul-2019 & ESPD: $6 \mathrm{M} 18 \mathrm{D}$ & GSLV & Mark-III-M1 & SHAR & INDIA \\
\hline 155. & Vikram/Pragyan & & \begin{tabular}{|l} 
Lander/Rover \\
\end{tabular} & ISRO & 22-Jul-2019 & 06-Sep-2019 & GSLV & Mark-III-M1 & SHAR & INDIA \\
\hline
\end{tabular}


Table-2 Spacecraft Parameters of Lunar Missions

\begin{tabular}{|c|c|c|c|c|c|c|c|c|c|c|c|}
\hline \multirow[t]{2}{*}{ S.No } & \multirow[t]{2}{*}{ Spacecraft } & \multirow[t]{2}{*}{ Distinct Name } & \multirow[t]{2}{*}{$\begin{array}{c}\text { No. of } \\
\text { Instruments }\end{array}$} & \multirow[t]{2}{*}{ Spacecraft Mass } & \multirow[t]{2}{*}{ Launch Mass } & \multirow{2}{*}{$\begin{array}{l}\text { Approx. } \\
\text { Spacecraft } \\
\text { Mass }\end{array}$} & \multirow{2}{*}{$\begin{array}{c}\text { Approx. } \\
\text { Launch } \\
\text { Mass }\end{array}$} & \multirow[t]{2}{*}{$\begin{array}{l}\text { Mission } \\
\text { Duration }\end{array}$} & \multicolumn{2}{|c|}{$\begin{array}{c}\text { Mission } \\
\text { Intermission } \\
\text { between }\end{array}$} & \multirow[t]{2}{*}{$\begin{array}{c}\text { Mission } \\
\text { Degradation }\end{array}$} \\
\hline & & & & & & & & & L-Date & D-Date & \\
\hline 01. & Pioneer 0 & Able-1 & 03 & 38 & 38 & 38 & 38 & 0.00089 & 37 & 37 & 01 \\
\hline 02. & Luna E-1 No.1 & & 05 & 156 & 360 & 156 & 360 & 0.00108 & 18 & 20 & 01 \\
\hline 03. & Pioneer 1 & Able-2 & 05 & 34.2 & 38.3 & 36.25 & 38.3 & 1.8 & 01 & 01 & 02 \\
\hline 04. & Luna E-1.No.2 & & 05 & 156 & 360 & 156 & 360 & 0.0012 & 27 & 27 & 01 \\
\hline 05. & Pioneer 2 & Able-3 & 06 & 39.2 & 39.5 & 39.5 & 39.2 & 0.03125 & 26 & 26 & 01 \\
\hline 06. & Luna E-1.No.3 & & 05 & 156 & 360 & 156 & 360 & 0.00284 & 02 & 03 & 01 \\
\hline 07. & Pioneer 3 & & 02 & 5.87 & 5.87 & 5.87 & 5.87 & 1.59 & 27 & 29 & 02 \\
\hline 08. & Mechta /Luna 1 & Luna E-1.No.4 & 05 & 205 & 360 & 181.15 & 282.5 & 2.58 & 60 & 60 & 03 \\
\hline 09. & Pioneer 4 & & 02 & 6.1 & 7.0 & 6.55 & 6.55 & 3.42 & 107 & 104 & 03 \\
\hline 10. & Luna E-1A.No.1 & Luna E-1A.No.5 & 05 & 156 & 390 & 156 & 390 & 0.00186 & 86 & 88 & 01 \\
\hline 11. & Luna 2 & Luna E-1A.No.2 & 05 & 205 & 390 & 180.5 & 297.5 & 1.46 & 12 & 12 & 02 \\
\hline 12. & Pioneer P-1 & & 10 & 25.3 & 88.4 & 25.3 & 88.4 & - & 10 & 26 & 02 \\
\hline 13. & Luna 3 & Luna E-2A.No.1 & 03 & 278 & 278.5 & 278.25 & 278.25 & 18 & 53 & 35 & 18 \\
\hline 14. & Pioneer P-3 & Able-IVB & 10 & 168.7 & 169 & 169 & 168.70 & 0.0012 & 141 & 141 & 01 \\
\hline 15. & Luna 1960A & Luna E-3.No.1 & 03 & 279 & 280 & 279.5 & 280 & 0.00844 & 04 & 04 & 01 \\
\hline 16. & Luna 1960B & Luna E-3.No.2 & 03 & 279 & 280 & 279 & 280 & 0.000116 & 159 & 159 & 01 \\
\hline 17. & Pioneer P-30 & Able-VA & 09 & 175.5 & 176 & 175.5 & 175.5 & 0.012 & 81 & 81 & 01 \\
\hline 18. & Pioneer P-31 & Able-VB & 10 & 175.5 & 176 & 176 & 175.5 & 0.000787 & 251 & 258 & 01 \\
\hline 19. & Ranger 1 & $\mathrm{P}-32$ & 07 & 304 & 306 & 306.18 & 306.2 & 7 & 87 & 81 & 07 \\
\hline 20. & Ranger 2 & P-33 & 08 & 304 & 306 & 306.18 & 304 & 2 & 69 & 75 & 02 \\
\hline 21. & Ranger 3 & P-34 & 04 & 188 & 336 & 259 & 336 & 1.4 & 87 & 83 & 02 \\
\hline 22. & Ranger 4 & $\mathrm{P}-35$ & 04 & 188 & 336 & 259.56 & 336 & 2.67 & 178 & 178 & 03 \\
\hline 23. & Ranger 5 & P-36 & 04 & 203 & 351 & 272.73 & 351 & 0.33 & 78 & 82 & 01 \\
\hline 24. & Sputnik 25 & Luna E-6.No.2 & 02 & 1500 & 2500 & 15. & 2500 & 0.045 & 30 & 23 & 07 \\
\hline 25. & Luna E-6.No.3 & Luna E-6.No.3 & 02 & 500 & 1420 & 1350 & 1420 & 0.00122 & 58 & 71 & 01 \\
\hline 26. & Luna 4 & Luna E-6.No.4 & 02 & 500 & 1420 & 925 & 1422 & 12 & 223 & 213 & 14 \\
\hline 27. & Kosmos 21 & Cosmos 21 & 09 & 800 & 890 & 800 & 890 & - & 80 & 80 & 03 \\
\hline 28. & Ranger 6 & P-53 & 01 & 172 & 381 & 276.5 & 372.85 & 2.74 & 52 & 48 & 03 \\
\hline 29. & Luna 1964A & Luna E-6.No.6 & 02 & 500 & 1420 & 1420 & 1422 & 0.00566 & 30 & 30 & 01 \\
\hline 30. & Luna E-6.No.5 & Luna E-6.No.5 & 02 & 500 & 1420 & 1420 & 1422 & 0.00394 & 99 & 102 & 01 \\
\hline 31. & Ranger 7 & $\mathrm{P}-54$ & 01 & 365 & 365 & 362.5 & 365.35 & 2.73 & 204 & 204 & 03 \\
\hline 32. & Ranger 8 & Ranger-C & 01 & 366 & 368 & 726 & 367.5 & 2.70 & 23 & 25 & 03 \\
\hline 33. & Kosmos 60 & Luna E-6.No.9 & 02 & 1600 & 6530 & 1600 & 6530 & - & 09 & 07 & 05 \\
\hline 34. & Ranger 9 & Ranger-D & 01 & 360 & 368 & 363.5 & 367.5 & 2.69 & 20 & 17 & 03 \\
\hline 35. & Luna 1965A & Luna E-6.No.8 & 02 & 1422 & 1470 & 1470 & 1422 & - & 29 & 32 & 01 \\
\hline 36. & Luna 5 & Luna E-6.No.10 & 02 & 300 & 1476 & 888 & 1422.5 & 3 & 30 & 37 & 03 \\
\hline 37. & Luna 6 & Luna E-6.No.7 & 02 & 280 & 1442 & 861 & 1441 & 10 & 40 & 258 & 10 \\
\hline 38. & Zond 3 & 3MV-4.No.3 & 06 & 960 & 960 & 960 & 960 & - & 78 & 147 & 228 \\
\hline 39. & Luna 7 & Luna E-6.No.11 & 02 & 300 & 1504 & 903 & 1505 & 3 & 60 & 60 & 03 \\
\hline 40. & Luna 8 & Luna E-6.No.12 & 02 & 350 & 1550 & 951 & 1551 & 3 & 59 & 62 & 03 \\
\hline 41. & Luna 9 & Luna E-6.No.13 & 02 & 300 & 1580 & 200 & 1581.5 & 6.46 & 29 & 25 & 06 \\
\hline
\end{tabular}




\begin{tabular}{|c|c|c|c|c|c|c|c|c|c|c|c|}
\hline S.No & Spacecraft & Distinct Name & $\begin{array}{c}\text { No. of } \\
\text { Instruments }\end{array}$ & Spacecraft Mass & Launch Mass & $\begin{array}{l}\text { Approx. } \\
\text { Spacecraft } \\
\text { Mass }\end{array}$ & $\begin{array}{c}\text { Approx. } \\
\text { Launch } \\
\text { Mass }\end{array}$ & $\begin{array}{l}\text { Mission } \\
\text { Duration }\end{array}$ & $\begin{array}{r}\text { Mis } \\
\text { Intern } \\
\text { betv } \\
\text { L-Date } \\
\end{array}$ & $\begin{array}{l}\text { ion } \\
\text { ission } \\
\text { een } \\
\text { D-Date } \\
\end{array}$ & $\begin{array}{c}\text { Mission } \\
\text { Degradation }\end{array}$ \\
\hline 42. & Kosmos 111 & Luna E-6S.No.204 & 07 & 1580 & 6459 & 1580 & 6459 & 2 & 30 & 88 & 02 \\
\hline 43. & Luna 10 & Luna E-6S.No.206 & 07 & 245 & 1582 & 172.5 & 1582 & 60 & 30 & 30 & 60 \\
\hline 44. & Luna 1966A & & - & 280 & 1600 & 940 & 1600 & - & 30 & 252 & 01 \\
\hline 45. & Surveyor 1 & Surveyor-A & 01 & 270 & 995 & 281 & 990.6 & 220 & 32 & 3545 & 222 \\
\hline 46. & Explorer 33 & AIMP-D & 05 & 57 & 94 & 75.2 & 153 & 1876 & 40 & 3615 & 3734 \\
\hline 47. & Lunar Orbiter 1 & Luna Orbiter-A & 03 & 261 & 385 & 323.3 & 382.8 & 78.19 & 11 & 28 & 79.7 \\
\hline 48. & Luna 11 & E-6LF.No.101 & 07 & 1308 & 1640 & 1474 & 1640 & 38 & 30 & 08 & 41.2 \\
\hline 49. & Surveyor 2 & Surveyor-B & 01 & 292 & 1004 & 292 & 999.6 & 2.62 & 32 & 118 & 03 \\
\hline 50. & Luna 12 & E-6LF.No.102 & 07 & 1288 & 1620 & 1288 & 1620 & - & 15 & 265 & 89 \\
\hline 51. & Lunar Orbiter 2 & Luna Orbiter-B & 03 & 261 & 385 & 261 & 382.8 & 339 & 45 & 287 & 339 \\
\hline 52. & Luna 13 & E-6M.No.205 & 05 & 300 & 1620 & 300 & 1620 & 6.83 & 37 & 30 & 07 \\
\hline 53. & Apollo 1 & & - & & 20000 & - & 20000 & 0 & 09 & 255 & -- \\
\hline 54. & Lunar Orbiter 3 & Luna Orbiter-C & 03 & 270 & 387 & 270 & 387.3 & 246 & 33 & 201 & 246 \\
\hline 55. & Kosmos 146 & 7K-L1.No.2P & - & 2800 & 5017 & 2800 & 5348.5 & 8 & 29 & 19 & 12 \\
\hline 56. & Kosmos 154 & 7K-L1.No.3P & - & 5017 & 5600 & 5017 & 5600 & 2 & 09 & 23 & 02 \\
\hline 57. & Surveyor 3 & Surveyor-C & 02 & 296 & 1036 & 299 & 1031 & 16 & 17 & 156 & 16 \\
\hline 58. & Lunar Orbiter 4 & Luna Orbiter-D & 03 & 270 & 389 & 270 & 387.3 & 180 & 13 & 36 & 155 \\
\hline 59. & Kosmos 159 & E6LS.No.111 & - & 283 & 5600 & 283 & 5600 & - & 58 & 117 & 178 \\
\hline 60. & Surveyor 4 & Surveyor-D & 03 & 299 & 1042 & 299 & 1040.5 & 2.58 & 05 & 2169 & 03 \\
\hline 61. & Explorer 35 & AIMP-E & 06 & 67 & 104 & 67 & 85.65 & 2167 & 13 & 1971 & 2167 \\
\hline 62. & Lunar Orbiter 5 & Luna Orbiter-E & 03 & 270 & 392 & 270 & 388.8 & 183 & 38 & 45 & 183 \\
\hline 63. & Surveyor 5 & Surveyor-E & 04 & 289 & 1006 & 296 & 1006 & 101 & 19 & 81 & 100 \\
\hline 64. & Zond 1967A & Soyuz 7K-L1.No.4L & - & 5375 & 5390 & 5375 & 5390 & 0.00118 & 41 & 78 & 01 \\
\hline 65. & Surveyor 6 & Surveyor-F & 04 & 289 & 1006 & 294.6 & 1006.5 & 37 & 15 & 22 & 37 \\
\hline 66. & Zond 1967B & Soyuz 7L-L1.No.5L & - & 5375 & 5390 & 5375 & 5390 & 0.0015 & 46 & 91 & 01 \\
\hline 67. & Surveyor 7 & Surveyor-G & 04 & 305 & 1038 & 305.35 & 1038.5 & 45 & 31 & 14 & 45 \\
\hline 68 & Luna 1968A & Luna E-6LS.No.112 & - & 5375 & 5600 & 5375 & 5600 & 0.00607 & 24 & 29 & 01 \\
\hline 69. & Zond 4 & 7K-L1.No.6L & - & 2800 & 5680 & 4087.5 & 5410 & - & 36 & 109 & 05 \\
\hline 70. & Luna 14 & E-6LS.No.113 & - & 1368 & 1700 & 1368 & 1700 & 75 & 15 & 63 & 78 \\
\hline 71. & Zond 1968A & Soyuz 7K-L1.7L & - & 5375 & 5600 & 5375 & 5375 & 0.003125 & 145 & 152 & 01 \\
\hline 72. & Zond 5 & 7K-L1.No.9L & 03 & 2800 & 5680 & 2800 & 5527.5 & 6.67 & 27 & 57 & 07 \\
\hline 73. & Apollo 7 & & - & 5175 & 16519 & 5175 & 16519 & 10.84 & 30 & 31 & 11 \\
\hline 74. & Zond 6 & 7K-L1.No.12L & 05 & 2800 & 5680 & 4087.5 & 5527.5 & 7 & 41 & 26 & 07 \\
\hline 75. & Apollo 8 & & - & 4979 & 28870 & 8326 & 26201 & 6.125 & 30 & 40 & 07 \\
\hline 76. & Zond 1969A & Soyuz 7K-L1.No.13L & - & 5375 & 5600 & 5375 & 5600 & 0.00579 & 30 & 24 & 01 \\
\hline 77. & Luna 1969A & Luna E-8.No.201 & 07 & 5590 & 5700 & 5590 & 5700 & 0.00059 & 02 & 30 & 01 \\
\hline 78. & Zond L1S-1 & Soyuz 7K-L1S.No.3S & - & 6900 & 6900 & 6900 & 6900 & 0.00081 & 10 & 20 & 01 \\
\hline 79. & Apollo 9 & LM-Spider & - & 5032 & 43,196 & 5032 & 43196 & 10.042 & 43 & 33 & -- \\
\hline 80. & Luna 1969B & & - & 5375 & 5700 & 5375 & 5700 & - & 33 & 53 & 01 \\
\hline 81. & Apollo 10 & LM-Snoopy & - & 4945 & 44576 & 4945 & 44576 & 8.00234 & 27 & 41 & 08 \\
\hline 82. & Luna 1969C & Luna E-8-5.No.402 & 03 & 5600 & 5600 & 5600 & 5600 & - & 19 & 19 & 01 \\
\hline 83. & Zond L1S-2 & Soyuz 7L-L1S.No.5L & - & 6900 & 6900 & 6900 & 6900 & 0.000118 & 10 & 18 & 01 \\
\hline
\end{tabular}




\begin{tabular}{|c|c|c|c|c|c|c|c|c|c|c|c|}
\hline \multirow[t]{2}{*}{ S.No } & \multirow[t]{2}{*}{ Spacecraft } & \multirow{2}{*}{ Distinct Name } & \multirow{2}{*}{$\begin{array}{c}\text { No. of } \\
\text { Instruments }\end{array}$} & \multirow{2}{*}{ Spacecraft Mass } & \multirow[t]{2}{*}{ Launch Mass } & \multirow{2}{*}{$\begin{array}{l}\text { Approx. } \\
\text { Spacecraft } \\
\text { Mass }\end{array}$} & \multirow{2}{*}{$\begin{array}{c}\text { Approx. } \\
\text { Launch } \\
\text { Mass }\end{array}$} & \multirow{2}{*}{$\begin{array}{c}\text { Mission } \\
\text { Duration }\end{array}$} & \multicolumn{2}{|c|}{$\begin{array}{c}\text { Mission Intermission } \\
\text { between }\end{array}$} & \multirow{2}{*}{$\begin{array}{c}\text { Mission } \\
\text { Degradation }\end{array}$} \\
\hline & & & & & & & & & L-Date & D-Date & \\
\hline 84. & Luna 15 & E-8-5.No.401 & 03 & 1360 & 5700 & 3480 & 5700 & 8 & 03 & 03 & 08 \\
\hline 85. & Apollo 11 & LM-Eagle & - & 4932 & 45702 & 4932 & 45702 & 8.138 & 22 & 21 & 09 \\
\hline 86. & Zond 7 & 7K-L1.No.11L & 03 & 2800 & 5680 & 2800 & 5829.5 & 6.67 & 47 & 44 & 07 \\
\hline 87. & Kosmos 300 & E-8-5.No.403 & 03 & 5600 & 5700 & 5600 & 5700 & - & 29 & 27 & 04 \\
\hline 88. & Kosmos 305 & E-8-5.No.404 & 03 & 5600 & 5700 & 5600 & 5700 & - & 23 & 31 & 02 \\
\hline 89. & Apollo 12 & LM-Intrepid & - & 5010 & 45870 & 5010 & 45870 & 10.20 & 84 & 74 & 10 \\
\hline 90. & Luna 1970A & Luna E-8-5.No.405 & 03 & 5600 & 5700 & 5600 & 5700 & - & 64 & 70 & 01 \\
\hline 91. & Luna 1970B & & - & 5600 & 5700 & 5600 & 5700 & - & 154 & 160 & 06 \\
\hline 92. & Apollo 13 & LM-Aquarius & - & 5050 & 45931 & 5050 & 45931 & 5.954 & 38 & 33 & 12 \\
\hline 93. & Luna 16 & E-8-5.No.406 & 03 & 1360 & 5700 & 3480 & 5700 & 12 & 21 & 21 & 07 \\
\hline 94. & Zond 8 & 7K-L1.No.14L & 02 & 5375 & 5700 & 5375 & 5375 & 07 & 82 & 84 & 308 \\
\hline 95. & Luna 17 & E-8.No.203 & 07 & 1124 & 5700 & 3362 & 4522 & 308 & 147 & 138 & 10 \\
\hline 96. & Apollo 14 & LM-Antares & - & 5208 & 46305 & 5208 & 46305 & 9.00136 & 29 & 788 & 01 \\
\hline 97. & Soyuz 7K-LOK & 7K-L1E.No.1 & - & 6698 & 9850 & 6698 & 9850 & - & 29 & 712 & 13 \\
\hline 98. & Apollo 15 & LM-Falcon & - & 5321 & 48599 & 5321 & 48599 & 12.30 & 38 & 712 & 759 \\
\hline 99. & PFS-1 & & 03 & 36 & 36 & 36 & 36 & 537 & 26 & 39 & 09 \\
\hline 100. & Luna 18 & E-8-5.No.407 & 04 & 1360 & 5700 & 3480 & 5725 & 09 & 139 & 128 & 388 \\
\hline 101. & Luna 19 & E-8LS.No.202 & 07 & 3000 & 3800 & 4300 & 4750 & 388 & 62 & 62 & 11 \\
\hline 102. & Luna 20 & E-8-5.No.408 & 04 & 1360 & 5727 & 3480 & 5134.5 & 11 & 62 & 32 & 12 \\
\hline 103. & Apollo 16 & LM-Orion & - & 5441 & 48637 & 5441 & 48637 & 11.077 & 78 & 35 & 43 \\
\hline 104. & PFS-2 & & 03 & 36 & 36 & 36 & 36 & 34 & 143 & 143 & 01 \\
\hline 105. & Soyuz 7K-LOK & Soyuz 7K-LOK.No.1 & - & 2505 & 6316 & 2505 & 6316 & - & 14 & 26 & 01 \\
\hline 106. & Soyuz 7K-LOK & 7K-LOK.No.6A & - & 9500 & 9850 & 9500 & 9850 & 0.00124 & 32 & 27 & 13 \\
\hline 107. & Apollo 17 & LM-Challenger & - & 5500 & 48607 & 5500 & 48607 & 12.578 & 153 & 1688 & 08 \\
\hline 108. & Luna 21 & E-8.No.204 & 10 & 4850 & 5950 & 2987 & 4647 & 08 & 146 & 890 & 1542 \\
\hline 109. & Explorer 49 & RAE-B & 05 & 330.2 & 328 & 330.2 & 328 & 1520 & 207 & 230 & 508 \\
\hline 110. & Mariner 10 & & 07 & 502.9 & 502.9 & 502.9 & 502.9 & 508 & 162 & 366 & 521 \\
\hline 111. & Luna22 & E-8LS.No.206 & 07 & 3000 & 3800 & 4000 & 4750 & 521 & 353 & 348 & 12 \\
\hline 112. & Luna 23 & E-8-5M.No.410 & 04 & 1360 & 5800 & 3480 & 4930.5 & 12 & 298 & 304 & 07 \\
\hline 113. & Luna 1975A & Luna E-8-5.No.412 & 04 & 5600 & 5800 & 5600 & 5800 & 07 & 733 & 7561 & 13 \\
\hline 114. & Luna 24 & E-8-5M.No.413 & 04 & 1360 & 5800 & 1360 & 5750 & 13 & 4085 & 2331 & 6841 \\
\hline 115. & ISEE-3 & Explorer 59/ICE & 13 & 389 & 479 & 389.5 & 479 & 6840 & 98 & 3816 & 5086 \\
\hline 116. & Galileo & & 10 & 1297 & 2562 & 1590.5 & 2433 & 5086 & 98 & 760 & 1172 \\
\hline 117. & Hiten & MUSES-A & 01 & 143 & 197 & 146 & 191.2 & 1172 & 912 & 760 & 1172 \\
\hline 118. & Hagoromo & & 01 & 08 & 08 & 08 & 12 & 1172 & 550 & - & - \\
\hline 119. & Geotail & & - & 640 & 980 & 640 & 994.5 & - & 280 & 8164 & - \\
\hline 120. & Clementine & DSPSE & 09 & 183 & 424 & 205 & 419 & - & 29 & 27 & 04 \\
\hline 121. & WIND & GGS & 12 & 895 & 1250 & 922.5 & 1222.5 & 115 & 1079 & - & 470 \\
\hline 122. & Cassini & Huygens & 11 & 2523 & 5712 & 2324 & 5483 & 7274 & 70 & 5555 & 7275 \\
\hline 123. & HGS-1 & Asiasat-3/PAS-22 & - & 1822 & 3480 & 2611 & 3472.5 & 1644 & 14 & 1066 & 1650 \\
\hline 124. & Lunar Prospector & Discovery 3 & 06 & 158 & 296 & 142 & 295.5 & 570 & 542 & 1592 & 570 \\
\hline 125. & Nozomi & Planet-B & 14 & 262 & 540 & 260 & 538 & 1980 & 1093 & 2507 & 1985 \\
\hline 126. & WMAP & & 05 & 768 & 840 & 765.5 & 837.5 & 3320 & 819 & 1508 & 3400 \\
\hline 127. & SMART-1 & & 07 & 287 & 367 & 287 & 367 & 1070.26 & 1124 & 3673 & 1072 \\
\hline
\end{tabular}




\begin{tabular}{|c|c|c|c|c|c|c|c|c|c|c|c|}
\hline S.No & Spacecraft & Distinct Name & $\begin{array}{c}\text { No. of } \\
\text { Instruments }\end{array}$ & Spacecraft Mass & Launch Mass & $\begin{array}{c}\text { Approx. } \\
\text { Spacecraft } \\
\text { Mass }\end{array}$ & $\begin{array}{c}\text { Approx. } \\
\text { Launch } \\
\text { Mass }\end{array}$ & $\begin{array}{l}\text { Mission } \\
\text { Duration }\end{array}$ & $\begin{array}{r}\text { Mis } \\
\text { Intern } \\
\text { betv } \\
\text { L-Date } \\
\end{array}$ & $\begin{array}{l}\text { ion } \\
\text { ission } \\
\text { een } \\
\text { D-Date } \\
\end{array}$ & $\begin{array}{c}\text { Mission } \\
\text { Degradation }\end{array}$ \\
\hline 128. & STEREO-A & & 04 & 560 & 620 & 553.5 & 619.5 & 4892 & 115 & - & - \\
\hline 129. & STEREO-B & & 04 & 560 & 620 & 553.5 & 619.5 & 3612 & 115 & 2662 & 3621 \\
\hline 130. & ARTEMIS-P1 & & 07 & 76 & 630 & 76.5 & 377.5 & 4780 & 209 & - & - \\
\hline 131. & ARTEMIS-P2 & & 07 & 76 & 630 & 76.5 & 377.5 & 4780 & 209 & - & - \\
\hline 132. & SELENE & Kaguya & 13 & 1984 & 2914 & 1852 & 2899.5 & 640 & 249 & 118 & - \\
\hline 133. & Okina & RSAT & 01 & 45 & 45 & 45 & 45 & - & 249 & 137 & 635 \\
\hline 134. & Ouna & VRAD & 01 & 53 & 35 & 53 & 53 & - & 249 & 120 & 517 \\
\hline 135. & Chang'e 1 & & 06 & 1000 & 2350 & 1000 & 2351 & 490 & 363 & 180 & 654 \\
\hline 136. & Chandrayaan 1 & & 11 & 560 & 1380 & 528 & 1380 & 306.8 & 603 & 287 & 494 \\
\hline 137. & MIP & Moon Impact Probe & 01 & 29 & 34 & 31.5 & 29 & - & 603 & 329 & 310 \\
\hline 138. & LRO & & 07 & 1018 & 1916 & 1018 & 1916 & 3945 & 470 & - & 21 \\
\hline 139. & LCROSS & & 09 & 585 & 2305 & 603 & 2277 & 20.59 & 470 & 1165 & - \\
\hline 140. & Chang'e 2 & & 04 & 1000 & 2480 & 1000 & 2480 & 3456 & 344 & - & 113 \\
\hline 141. & $\mathrm{Ebb}$ & GRAIL-A & 03 & 132.6 & 307 & 166.8 & 307 & 462.38 & 728 & 487 & 3456 \\
\hline 142. & Flow & GRAIL-B & 03 & 132.6 & 307 & 166.8 & 307 & 462.38 & 728 & 487 & 346 \\
\hline 143. & LADEE & & 03 & 248 & 383 & 248.1 & 383 & 223 & 85 & 317 & 346 \\
\hline 144. & Chang'e 3 & & 04 & 1200 & 3800 & 1200 & 3790 & 959 & 326 & - & 223 \\
\hline 145. & Yutu & & 03 & 140 & 140 & 140 & 1970 & 42 & 326 & 121 & 959 \\
\hline 146. & Chang'e 5-T1 & & 02 & 1000 & 2480 & 667.5 & 2347.5 & 1973 & 1273 & 11 & 485 \\
\hline 147. & MMMM & 4M Mission & 02 & 14 & 14 & 14 & 14 & 19 & 1273 & 1289 & 1973 \\
\hline 148. & TESS & & 04 & 317 & 362 & 317 & 362 & 700 & 32 & - & 19 \\
\hline 149. & Queqiao & Chang'e 4 Relay & 02 & 325 & 425 & 325 & 425 & - & 201 & - & - \\
\hline 150. & Longjiang 1 & DSWLP-A1/LO-93 & - & 45 & 45 & 46 & 45 & - & 201 & 436 & - \\
\hline 151. & Longjiang 2 & DSWLP-A2/LO-94 & - & 45 & 45 & 45 & 45 & - & 201 & 118 & 01 \\
\hline 152. & Chang'e 4 & & 09 & 1200 & 3780 & 1200 & 3780 & 440 & 77 & - & 437 \\
\hline 153. & Beresheet & & 02 & 150 & 585 & 150 & 585 & 47.7 & 150 & 155 & 440 \\
\hline 154. & Chandrayaan 2 & & 08 & 1308 & 3850 & 995 & 3850 & - & - & - & 48 \\
\hline 155. & Vikram/Pragyan & & 06 & 626 & 1471 & 612.5 & 1471 & - & - & - & 46 \\
\hline
\end{tabular}


Table-3 Lunar Exploration Missions and their Objectives

\begin{tabular}{|c|c|c|c|c|c|c|c|}
\hline S.No & Spacecraft & Distinct Name & Objective of the Mission & S.No & Spacecraft & Distinct Name & Objective of the Mission \\
\hline 01. & Pioneer 0 & Able-1 & Launch Probe into Deep Space & 41. & Luna 9 & Luna E-6.No.13 & Soft-Landing attempt \\
\hline 02. & Luna E-1 No.1 & & Intended to Impact the Moon & 42. & Kosmos 111 & Luna E-6S.No.204 & Orbit around the Moon \\
\hline 03. & Pioneer 1 & Able-2 & Intended to Orbit around Moon & 43. & Luna 10 & Luna E-6S.No.206 & Orbit around the Moon \\
\hline 04. & Luna E-1.No.2 & & Intended to Impact the Moon & 44. & Luna 1966A & & Orbit around the Moon \\
\hline 05. & Pioneer 2 & Able-3 & To probe Cislunar Space & 45. & Surveyor 1 & Surveyor-A & Lunar Soft-Landing attempt \\
\hline 06. & Luna E-1.No.3 & & Intended to Impact the Moon & 46. & Explorer 33 & AIMP-D & Orbit around the Moon \\
\hline 07. & Pioneer 3 & & To Orbit around the Moon & 47. & Lunar Orbiter 1 & Luna Orbiter-A & Orbit around the Moon \\
\hline 08. & Mechta /Luna 1 & Luna E-1.No.4 & Intended to Impact the Moon & 48. & Luna 11 & E-6LF.No.101 & Orbit around the Moon \\
\hline 09. & Pioneer 4 & & Flyby around the Moon & 49. & Surveyor 2 & Surveyor-B & Lunar Soft-Landing attempt \\
\hline 10. & Luna E-1A.No.1 & Luna E-1A.No.5 & Intended to Impact the Moon & 50. & Luna 12 & E-6LF.No.102 & Orbit around the Moon \\
\hline 11. & Luna 2 & Luna E-1A.No.2 & Intended to Impact the Moon & 51. & Lunar Orbiter 2 & Luna Orbiter-B & Orbit around the Moon \\
\hline 12. & Pioneer P-1 & & Heavy Instrumented Probe in Lunar Orbit & 52. & Luna 13 & E-6M.No.205 & Lunar Soft-Landing attempt \\
\hline 13. & Luna 3 & Luna E-2A.No.1 & Photograph far side of the Moon & 53. & Apollo 1 & & Manned Moon Mission \\
\hline 14. & Pioneer P-3 & Able-IVB & Heavy Instrumented Probe in Lunar Orbit & 54. & Lunar Orbiter 3 & Luna Orbiter-C & Orbit around the Moon \\
\hline 15. & Luna 1960A & Luna E-3.No.1 & Flyby around the Moon in Cislunar Orbit & 55. & Kosmos 146 & 7K-L1.No.2P & Flyby \& Return to Earth Test \\
\hline 16. & Luna 1960B & Luna E-3.No.2 & Flyby around the Moon in Cislunar Orbit & 56. & Kosmos 154 & 7K-L1.No.3P & Test Spacecraft \\
\hline 17. & Pioneer P-30 & Able-VA & Heavy Instrumented Probe in Lunar Orbit & 57. & Surveyor 3 & Surveyor-C & Lunar Soft-landing attempt \\
\hline 18. & Pioneer P-31 & Able-VB & Heavy Instrumented Probe in Lunar Orbit & 58. & Lunar Orbiter 4 & Luna Orbiter-D & Orbit around the Moon \\
\hline 19. & Ranger 1 & $\mathrm{P}-32$ & Test Performance Probe & 59. & Kosmos 159 & E6LS.No.111 & Achieve high elliptical Earth Orbit \\
\hline 20. & Ranger 2 & $\mathrm{P}-33$ & Test Performance Probe & 60. & Surveyor 4 & Surveyor-D & Lunar Soft-landing attempt \\
\hline 21. & Ranger 3 & $\mathrm{P}-34$ & First attempt to land on the Moon & 61. & Explorer 35 & AIMP-E & Orbit around the Moon \\
\hline 22. & Ranger 4 & $\mathrm{P}-35$ & Intended to Impact the Moon & 62. & Lunar Orbiter 5 & Luna Orbiter-E & Orbit around the Moon \\
\hline 23. & Ranger 5 & P-36 & Intended to Impact the Moon & 63. & Surveyor 5 & Surveyor-E & Lunar Soft-landing attempt \\
\hline 24. & Sputnik 25 & Luna E-6.No.2 & Soft-Landing attempt & 64. & Zond 1967A & Soyuz 7K-L1.No.4L & Perform Circumlunar Flyby \\
\hline 25. & Luna E-6.No.3 & Luna E-6.No.3 & Soft-Landing attempt & 65. & Surveyor 6 & Surveyor-F & Lunar Soft-landing attempt \\
\hline 26. & Luna 4 & Luna E-6.No.4 & Soft-Landing attempt & 66. & Zond 1967B & Soyuz 7L-L1.No.5L & Perform Circumlunar Flyby \\
\hline 27. & Kosmos 21 & Cosmos 21 & Deep Space Technology Test & 67. & Surveyor 7 & Surveyor-G & Lunar Soft-landing attempt \\
\hline 28. & Ranger 6 & $\mathrm{P}-53$ & Achieve Lunar Impact Trajectory & 68 & Luna 1968A & Luna E-6LS.No.112 & Orbit around the Moon \\
\hline 29. & Luna 1964A & Luna E-6.No.6 & Soft-Landing attempt & 69. & Zond 4 & 7K-L1.No.6L & Crewed Flyby Spacecraft Test \\
\hline 30. & Luna E-6.No.5 & Luna E-6.No.5 & Soft-Landing attempt & 70. & Luna 14 & E-6LS.No.113 & Orbit around the Moon \\
\hline 31. & Ranger 7 & $\mathrm{P}-54$ & Achieve Lunar Impact Trajectory & 71. & Zond 1968A & Soyuz 7K-L1.7L & Crewed Lunar Flyby Test \\
\hline 32. & Ranger 8 & Ranger-C & Obtain Close-up Image of Moon & 72. & Zond 5 & 7K-L1.No.9L & To perform Circumlunar Flyby \\
\hline 33. & Kosmos 60 & Luna E-6.No.9 & Soft-Landing attempt & 73. & Apollo 7 & & Crewed Orbital Flyby Test \\
\hline 34. & Ranger 9 & Ranger-D & Achieve Lunar Impact Trajectory & 74. & Zond 6 & 7K-L1.No.12L & Crewed Lunar Flyby Test \\
\hline 35. & Luna 1965A & Luna E-6.No.8 & Soft-Landing attempt & 75. & Apollo 8 & & Crewed Spaceflight Mission \\
\hline 36. & Luna 5 & Luna E-6.No.10 & Soft-Landing attempt & 76. & Zond 1969A & Soyuz 7K-L1.No.13L & Lunar Flyby Circumlunar Flight \\
\hline 37. & Luna 6 & Luna E-6.No.7 & Soft-Landing attempt & 77. & Luna 1969A & Luna E-8.No.201 & Lunar Landing Attempt \\
\hline 38. & Zond 3 & 3MV-4.No.3 & Flyby around the Moon & 78. & Zond L1S-1 & Soyuz 7K-L1S.No.3S & NI Launch Test \\
\hline 39. & Luna 7 & Luna E-6.No.11 & Soft-Landing attempt & 79. & Apollo 9 & LM-Spider & Landing on the Moon Test \\
\hline 40. & Luna 8 & Luna E-6.No.12 & Soft-Landing attempt & 80. & Luna 1969B & & Sample Return Attempt \\
\hline
\end{tabular}




\begin{tabular}{|c|c|c|c|c|c|c|c|}
\hline S.No & Spacecraft & Distinct Name & Objective of the Mission & S.No & Spacecraft & Distinct Name & Objective of the Mission \\
\hline 81. & Apollo 10 & LM-Snoopy & Crewed Spaceflight Mission & 121. & WIND & GGS & Flyby around the Moon \\
\hline 82. & Luna 1969C & Luna E-8-5.No.402 & Sample Return Attempt & 122. & Cassini & Huygens & Saturn/Gravity Assist \\
\hline 83. & Zond L1S-2 & Soyuz 7L-L1S.No.5L & N1 Launch Test & 123. & HGS-1 & Asiasat-3/PAS-22 & Gravity Assist Probe \\
\hline 84. & Luna 15 & E-8-5.No.401 & Lunar Sample Return Attempt & 124. & Lunar Prospector & Discovery 3 & Orbit around the Moon \\
\hline 85. & Apollo 11 & LM-Eagle & First Crewed Landing on the Moon & 125. & Nozomi & Planet-B & Gravity Assist Probe \\
\hline 86. & Zond 7 & 7K-L1.No.11L & To Perform Circumlunar Flyby & 126. & WMAP & & Gravity Assist Probe \\
\hline 87. & Kosmos 300 & E-8-5.No.403 & Lunar Sample Return Attempt & 127. & SMART-1 & & Orbit around the Moon \\
\hline 88. & Kosmos 305 & E-8-5.No.404 & Lunar Sample Return Attempt & 128. & STEREO-A & & Orbit around the Moon \\
\hline 89. & Apollo 12 & LM-Intrepid & Crewed Lunar Landing & 129. & STEREO-B & & Orbit around the Moon \\
\hline 90. & Luna 1970A & Luna E-8-5.No.405 & Lunar Sample Return Attempt & 130. & ARTEMIS-P1 & & To Study Earth's Magnetosphere \\
\hline 91. & Luna 1970B & & Orbit around the Moon & 131. & ARTEMIS-P2 & & To Study Earth's Magnetosphere \\
\hline 92. & Apollo 13 & LM-Aquarius & Crewed Lunar Landing Mission & 132. & SELENE & Kaguya & Orbit around the Moon \\
\hline 93. & Luna 16 & E-8-5.No.406 & Lunar Sample Return Attempt & 133. & Okina & RSAT & Orbit around the Moon \\
\hline 94. & Zond 8 & 7K-L1.No.14L & To Perform Circumlunar Flyby & 134. & Ouna & VRAD & Orbit around the Moon \\
\hline 95. & Luna 17 & E-8.No.203 & Lunar Roving Operations & 135. & Chang'e 1 & & Orbit around the Moon \\
\hline 96. & Apollo 14 & LM-Antares & Crewed Lunar Landing Mission & 136. & Chandrayaan 1 & & Orbit around the Moon \\
\hline 97. & Soyuz 7K-LOK & 7K-L1E.No.1 & Crewed Lunar Flyby Test & 137. & MIP & Moon Impact Probe & Impact on the Moon \\
\hline 98. & Apollo 15 & LM-Falcon & Crewed Lunar Landing Mission & 138. & LRO & & Orbit in an eccentric polar orbit \\
\hline 99. & PFS-1 & & Orbit around the Moon & 139. & LCROSS & & Explore water near polar region \\
\hline 100. & Luna 18 & E-8-5.No.407 & Lunar Sample Return Attempt & 140. & Chang'e 2 & & Orbit around the Moon \\
\hline 101. & Luna 19 & E-8LS.No.202 & Orbit around the Moon & 141. & Ebb & GRAIL-A & To determine Moon Interior Structure \\
\hline 102. & Luna 20 & E-8-5.No.408 & Lunar Sample Return Attempt & 142. & Flow & GRAIL-B & To determine Moon Interior Structure \\
\hline 103. & Apollo 16 & LM-Orion & Crewed Lunar Landing Mission & 143. & LADEE & & To Study Lunar Exosphere \\
\hline 104. & PFS-2 & & Orbit around the Moon & 144. & Chang'e 3 & & Landing and Roving Attempt \\
\hline 105. & Soyuz 7K-LOK & Soyuz 7K-LOK.No.1 & Orbit around the Moon & 145. & Yutu & & Landing and Roving Attempt \\
\hline 106. & Soyuz 7K-LOK & 7K-LOK.No.6A & Crewed Lunar Flyby Test & 146. & Chang'e 5-T1 & & Lunar Capsule Re-entry Test \\
\hline 107. & Apollo 17 & LM-Challenger & Crewed Lunar Landing Mission & 147. & MMMM & 4M Mission & Flyby around the Moon \\
\hline 108. & Luna 21 & E-8.No.204 & Lunar Roving Operations & 148. & TESS & & Search for Exoplanet \\
\hline 109. & Explorer 49 & RAE-B & Orbit around the Moon & 149. & Queqiao & Chang'e 4 Relay & Communication Relay for Chang'e 4 \\
\hline 110. & Mariner 10 & & Mercury/Venus/Lunar Flyby & 150. & Longjiang 1 & DSWLP-A1/LO-93 & Microsatellite Mission - Orbiter \\
\hline 111. & Luna22 & E-8LS.No.206 & Orbit around the Moon & 151. & Longjiang 2 & DSWLP-A2/LO-94 & Microsatellite Mission - Orbiter \\
\hline 112. & Luna 23 & E-8-5M.No.410 & Lunar Sample Return Attempt & 152. & Chang'e 4 & & Landing and Roving Attempt \\
\hline 113. & Luna 1975A & Luna E-8-5.No.412 & Lunar Sample Return Attempt & 153. & Beresheet & & Lunar Lander Demonstrator Probe \\
\hline 114. & Luna 24 & E-8-5M.No.413 & Lunar Sample Return Attempt & 154. & Chandrayaan 2 & & Orbit around the Moon \\
\hline 115. & ISEE-3 & Explorer 59/ICE & Lunar Flyby & 155. & Vikram/Pragyan & & Lunar Soft-Landing Attempt in South Pole \\
\hline 116. & Galileo & & Jupiter/Earth/Lunar Flyby & & & & \\
\hline 117. & Hiten & MUSES-A & Flyby around the Moon & & & & \\
\hline 118. & Hagoromo & & Lunar Orbit & & & & \\
\hline 119. & Geotail & & Study Dynamics of Magnetosphere & & & & \\
\hline 120. & Clementine & DSPSE & Orbit around the Moon & & & & \\
\hline
\end{tabular}


Table-4 Chronology of Failed Lunar Missions

\begin{tabular}{|c|c|c|c|c|c|c|c|c|c|c|}
\hline S.No & Spacecraft & Distinct Name & Type & Manufacturer & Launch Date & $\begin{array}{c}\text { End of Mission } \\
\text { Decay Date }\end{array}$ & Launcher & Launcher Type & Launch Site & Country \\
\hline 01. & Pioneer 0 & Able-1 & Orbiter & STL & 17-Aug-1958 & 17-Aug-1958 & Thor & DM-18-Able-1 & CC-LC-17A & US \\
\hline 02. & Luna E-1 No.1 & & Impactor & OKB-1 & 23-Sep-1958 & 23-Sep-1958 & Luna $8 \mathrm{~K} 72$ & S.No. B1-3 & Baikonur 1/5 & USSR \\
\hline 03. & Pioneer 1 & Able-2 & Orbiter & STL & 11-Oct-1958 & 13 -Oct-1958 & Thor & DM-18-Able-1 & CC-LC-17A & US \\
\hline 04. & Luna E-1.No.2 & & Impactor & OKB-1 & 12-Oct-1958 & 12-Oct-1958 & Luna 8 K72 & S.No. B1-4 & Baikonur $1 / 5$ & USSR \\
\hline 05. & Pioneer 2 & Able-3 & Orbiter & STL & 08-Nov-1958 & 08-Nov-1958 & Thor & DM-18-Able-1 & CC-LC-17A & US \\
\hline 06. & Luna E-1.No.3 & & Impactor & OKB-1 & 04-Dec-1958 & 04-Dec-1958 & Luna $8 \mathrm{~K} 72$ & S.No. B1-5 & Baikonur 1/5 & USSR \\
\hline 07. & Pioneer 3 & & Flyby & JPL & 06-Dec-1958 & 07-Dec-1958 & Juno & Version - II & CC-LC-05 & US \\
\hline 08. & Mechta / Luna 1 & Luna E-1.No.4 & Impactor & OKB-1 & 02-Jan-1959 & 05-Jan-1959 & Luna $8 \mathrm{~K} 72$ & S.No. B1-6 & Baikonur 1/5 & USSR \\
\hline 09. & Pioneer 4 & & Flyby & JPL & 03-Mar-1959 & 06-Mar-1959 & Juno & Version - II & CC-LC-05 & US \\
\hline 10. & Luna E-1A.No.1 & Luna E-1A.No.5 & Impactor & OKB-1 & 18-Jun-1959 & 18-Jun-1959 & Luna $8 \mathrm{~K} 72$ & S.No. L1-7 & Baikonur 1/5 & USSR \\
\hline 11. & Pioneer P-1 & & Orbiter & TRW-STL & 24-Sep-1959 & 26-Sep-1959 & Atlas-C & Able & CC-LC-12 & US \\
\hline 12. & Pioneer P-3 & Able-IVB & Orbiter & TRW-STL & 26-Nov-1959 & 26-Nov-1959 & Atlas-D & Able & CC-LC-14 & US \\
\hline 13. & Luna 1960A & Luna E-3.No.1 & Circumlunar & OKB-1 & 15-Apr-1960 & 15-Apr-1960 & Luna $8 \mathrm{~K} 72$ & S.No.L1-9 & Baikonur 1/5 & USSR \\
\hline 14. & Luna 1960B & Luna E-3.No.2 & Circumlunar & OKB-1 & 19-Apr-1960 & 19-Apr-1960 & Luna $8 \mathrm{~K} 72$ & S.No.L1-9A & Baikonur 1/5 & USSR \\
\hline 15. & Pioneer P-30 & Able-VA & Orbiter & TRW-STL & $25-$ Sep-1960 & 25-Sep-1960 & Atlas-D & Able & CC-LC-12 & US \\
\hline 16. & Pioneer P-31 & Able-VB & Orbiter & TRW-STL & 15-Dec-1960 & 15-Dec-1960 & Atlas-D & Able & CC-LC-12 & US \\
\hline 17. & Ranger 3 & P-34 & Impactor & JPL & 26-Jan-1962 & 02-Feb-1962 & Atlas LV-3 & Agena-B & CC-LC-12 & US \\
\hline 18. & Ranger 4 & P-35 & Impactor & JPL & 23-Apr-1962 & 26-Apr-1962 & Atlas LV-3 & Agena-B & CC-LC-12 & US \\
\hline 19. & Ranger 5 & P-36 & Impactor & JPL & 18 -Oct-1962 & 21-Oct-1962 & Atlas LV-3 & Agena-B215D-AA7 & CC-LC-12 & US \\
\hline 20. & Sputnik 25 & Luna E-6.No.2 & Lander & OKB-1 & 04-Jan-1963 & 11-Jan-1963 & Molniya-L & 8K78-S.No.T103-09 & Baikonur 1/5 & USSR \\
\hline 21. & Luna E-6.No.3 & Luna E-6.No.3 & Lander & OKB-1 & 03-Feb-1963 & 03-Feb-1963 & Molniya-L & 8K78-S.No.G103-10 & Baikonur 1/5 & USSR \\
\hline 22. & Luna 4 & Luna E-6.No.4 & Lander & OKB-1 & 02-Apr-1963 & 15-Apr-1963 & Molniya-L & $8 \mathrm{~K} 78 / \mathrm{E} 6 / \mathrm{G} 103-11$ & Baikonur 1/5 & USSR \\
\hline 23. & Kosmos 21 & Cosmos 21 & L1 Test & OKB-1 & 11-Nov-1963 & 14-Nov-1963 & Molniya-L & 8K78M.S.No.G103-18 & Baikonur 1/5 & USSR \\
\hline 24. & Ranger 6 & P-53 & Impactor & JPL & 30-Jan-1964 & 02-Feb-1964 & Atlas LV-3 & Agena-B/199D/AA8 & CC-LC-12 & US \\
\hline 25. & Luna 1964A & Luna E-6.No.6 & Lander & OKB-1 & 21-Mar-1964 & 21-Mar-1964 & Molniya-M & $8 \mathrm{~K} 78-\mathrm{M} / \mathrm{T} 15000-20$ & Baikonur 1/5 & USSR \\
\hline 26. & Luna E-6.No.5 & Luna E-6.No.5 & Lander & OKB-1 & 20-Apr-1964 & 20-Apr-1964 & Molniya-M & $8 \mathrm{~K} 78 \mathrm{~L} / \mathrm{R} 103-25$ & Baikonur 1/5 & USSR \\
\hline 27. & Kosmos 60 & Luna E-6.No.9 & Lander & OKB-1 & 12-Mar-1965 & 17-Mar-1965 & Molniya-M & $8 \mathrm{~K} 78 \mathrm{~L} / \mathrm{R} 103-26$ & Baikonur 1/5 & USSR \\
\hline 28. & Luna 1965A & Luna E-6.No.8 & Lander & OKB-1 & 10-Apr-1965 & 10-Apr-1965 & Molniya-M & $8 \mathrm{~K} 78-\mathrm{M} / \mathrm{R} 103-26$ & Baikonur 1/5 & USSR \\
\hline 29. & Luna 5 & Luna E-6.No.10 & Lander & OKB-1 & 09-Мay-1965 & 12-May-1965 & Molniya-M & $8 \mathrm{~K} 78-\mathrm{M} / \mathrm{U} 103-30$ & Baikonur 1/5 & USSR \\
\hline 30. & Luna 6 & Luna E-6.No.7 & Lander & OKB-1 & 08-Jun-1965 & 18-Jun-1965 & Molniya-M & $8 \mathrm{~K} 78-\mathrm{M} / \mathrm{U} 103-31$ & Baikonur 1/5 & USSR \\
\hline 31. & Luna 7 & Luna E-6.No.11 & Lander & OKB-1 & 04-Oct-1965 & 07-Oct-1965 & Molniya-M & $8 \mathrm{~K} 78-\mathrm{M} / \mathrm{U} 103-27$ & Baikonur 1/5 & USSR \\
\hline 32. & Luna 8 & Luna E-6.No.12 & Lander & OKB-1 & 03-Dec-1965 & 06-Dec-1965 & Molniya-M & $8 \mathrm{~K} 78-\mathrm{M} / \mathrm{U} 103-28$ & Baikonur 31/6 & USSR \\
\hline 33. & Kosmos 111 & Luna E-6S.No.204 & Orbiter & GSMZ-Lavochkin & 01-Mar-1966 & 03-Mar-1966 & Molniya-M & $8 \mathrm{~K} 78-\mathrm{M} / \mathrm{N} 103-41$ & Baikonur 31/6 & USSR \\
\hline 34. & Luna 1966A & & Orbiter & OKB-1 & 30-Apr-1966 & 30-Apr-1966 & Proton K/D & UR-500K & Baikonur 1/5 & USSR \\
\hline 35. & Explorer 33 & AIMP-D & Orbiter & NASA/GSFC & 01-Jul-1966 & 21-Sep-1976 & Delta & E1 & CC-LC-17A & US \\
\hline 36. & Lunar Orbiter 1 & Luna Orbiter-A & Orbiter & NASA/LARC & 10-Aug-1966 & 29-Oct-1966 & Atlas SLV-3 & Agena-D & CC-LC-13 & US \\
\hline 37. & Luna 11 & E-6LF.No.101 & Orbiter & GSMZ-Lavochkin & 21-Aug-1966 & 01-Oct-1966 & Molniya-M & $8 \mathrm{~K} 78-\mathrm{M}$ & Baikonur $31 / 6$ & USSR \\
\hline 38. & Surveyor 2 & Surveyor-B & Lander & NASA/JPL & 20-Sep-1966 & 23-Sep-1966 & Atlas LV-3C & Centaur-D & CC-LC-36A & US \\
\hline 39. & Kosmos 154 & 7K-L1.No.3P & L1 Test & OKB-1 & 08-Apr-1967 & 10-Apr-1967 & Proton K/D & UR-500K & Baikonur 81/36 & USSR \\
\hline 40. & Surveyor 4 & Surveyor-D & Lander & NASA/JPL & 14-Jul-1967 & 17-Jul-1967 & Atlas LV-3C & Centaur-D & CC-LC-36A & US \\
\hline 41. & Zond 1967A & Soyuz 7K-L1.No.4L & Flyby & OKB-1 & 27-Sep-1967 & 27-Sep-1967 & Proton K/D & S.No.229-01 & Baikonur $81 / 23$ & USSR \\
\hline 42. & Zond 1967B & Soyuz 7L-L1.No.5L & Flyby & OKB-1 & 22-Nov-1967 & 22-Nov-1967 & Proton K/D & S.No.230-01 & Baikonur 81/24 & USSR \\
\hline 43. & Luna 1968A & Luna E-6LS.No.112 & Orbiter & GSMZ-Lavochkin & 07-Feb-1968 & 07-Feb-1968 & Molniya-M & 8K78M.S.No.YG716-57 & Baikonur 1/5 & USSR \\
\hline 44. & Zond 4 & 7K-L1.No.6L & Deep Space & OKB-1 & 02-Mar-1968 & 07-Mar-1968 & Proton K/D & UR-500K & Baikonur 81/6 & USSR \\
\hline
\end{tabular}




\begin{tabular}{|c|c|c|c|c|c|c|c|c|c|c|}
\hline S.No & Spacecraft & Distinct Name & Type & Manufacturer & Launch Date & $\begin{array}{c}\text { End of Mission } \\
\text { Decay Date }\end{array}$ & Launcher & Launcher Type & Launch Site & Country \\
\hline 45. & Zond 1968A & Soyuz 7K-L1.7L & Flyby & OKB-1 & 22-Apr-1968 & 22-Apr-1968 & Soyuz & 7K-L1.S.No.7 & Baikonur 1/5 & USSR \\
\hline 46. & Zond 6 & 7K-L1.No.12L & Flyby & OKB-1 & 10-Nov-1968 & 17-Nov-1968 & Proton-K/D & $11 S 824$ & Baikonur 81/26 & USSR \\
\hline 47. & Zond 1969A & Soyuz 7K-L1.No.13L & Flyby & OKB-1 & 20-Jan-1969 & 20-Jan-1969 & Soyuz & 7K-L1.S.No.13 & Baikonur 81/24 & USSR \\
\hline 48. & Luna 1969A & Luna E-8.No.201 & Lander/Rover & GSMZ-Lavochkin & 19-Feb-1969 & 19-Feb-1969 & Proton K/D & S.No.239-01 & Baikonur 81/24 & USSR \\
\hline 49. & Zond L1S-1 & Soyuz 7K-L1S.No.3S & Orbiter & OKB-1 & 21-Feb-1969 & 21-Feb-1969 & N1 & SL-15 & Baikonur 110/38 & USSR \\
\hline 50. & Luna 1969B & & Sample Return & OKB-1 & 15-Apr-1969 & 15-Apr-1969 & Proton K/D & UR-500K & Baikonur 81/24 & USSR \\
\hline 51. & Luna 1969C & Luna E-8-5.No.402 & Sample Return & GSMZ-Lavochkin & 14-Jun-1969 & 14-Jun-1969 & Proton-K/D & UR-500K & Baikonur 81/24 & USSR \\
\hline 52. & Zond L1S-2 & Soyuz 7L-L1S.No.5L & Orbiter & OKB-1 & 03-Jul-1969 & 03-Jul-1969 & N1 & SL-15 & Baikonur 110/38 & USSR \\
\hline 53. & Luna 15 & E-8-5.No.401 & Sample Return & GSMZ-Lavochkin & 13-Jul-1969 & 21-Jul-1969 & Proton K/D & UR-500K/242-01 & Baikonur 81/24 & USSR \\
\hline 54. & Kosmos 300 & E-8-5.No.403 & Sample Return & GSMZ-Lavochkin & 23-Sep-1969 & 27-Sep-1969 & Proton K/D & UR-500K/244-01 & Baikonur 81/5 & USSR \\
\hline 55. & Kosmos 305 & E-8-5.No.404 & Sample Return & GSMZ-Lavochkin & 22-Oct-1969 & 24-Oct-1969 & Proton K/D & UR-500K/241-01 & Baikonur 81/5 & USSR \\
\hline 56. & Luna 1970A & Luna E-8-5.No.405 & Sample Return & GSMZ-Lavochkin & 06-Feb-1970 & 06-Feb-1970 & Proton K/D & S.No.247-01 & Baikonur 81/23 & USSR \\
\hline 57. & Luna 1970B & & Orbiter & GSMZ-Lavochkin & 19-Feb-1970 & 19-Feb-1970 & Proton K/D & SL-12/D-1.E & Baikonur 81/24 & USSR \\
\hline 58. & Soyuz 7K-LOK & 7K-L1E.No.1 & Circumlunar & OKB-1 & 27-Jun-1971 & 27-Jun-1971 & N1 & $\mathrm{N} 1$ & Baikonur 110/37 & USSR \\
\hline 59. & Luna 18 & E-8-5.No.407 & Sample Return & GSMZ-Lavochkin & 02-Sep-1971 & 11-Sep-1971 & Proton K/D & 8K82K /S.No.256-01 & Baikonur 81/24 & USSR \\
\hline 60. & Soyuz 7K-LOK & 7K-LOK.No.6A & Circumlunar & OKB-1 & 23-Nov-1972 & 23-Nov-1972 & N1 & N1 & Baikonur 110/37 & USSR \\
\hline 61. & Luna 23 & E-8-5M.No.410 & Sample Return & GSMZ-Lavochkin & 28-Oct-1974 & 09-Nov-1974 & Proton K/D & 8K82K/S.No.285-01 & Baikonur 81/24 & USSR \\
\hline 62. & Luna 1975A & Luna E-8-5.No.412 & Sample Return & GSMZ-Lavochkin & 16-Oct-1975 & 23-Oct-1975 & Proton K/D & SL-12/D-1.E & Baikonur 81/23 & USSR \\
\hline 63. & Hiten & MUSES-A & Flyby/Orbiter & JAXA & 24-Jan-1990 & 10-Apr-1993 & MU-3S11 & S.No.5-3S11 & Launch Complex M1 & JAPAN \\
\hline 64. & Hagoromo & & Orbiter & JAXA & 24-Jan-1990 & 10-Apr-1993 & $\mathrm{Mu}-3511$ & S.No.5-3S11 & Launch Complex M1 & JAPAN \\
\hline 65. & HGS-1 & Asiasat-3/PAS-22 & Gravity Assist & HUGHES & 24-Dec-1997 & 00-Jul-2002 & Proton-K & DM-3 & Baikonur & USSR \\
\hline 66. & Nozomi & Planet-B & Gravity Assist & JAXA & 03-Jul-1998 & 09-Dec-2003 & $\mathrm{M}-\mathrm{V}$ & $\mathrm{M}-\mathrm{V}$ & Launch Complex M5 & JAPAN \\
\hline 67. & Chandrayaan 1 & & Orbiter & ISRO & 21-Oct-2008 & 28-Aug-2009 & PSLV-XL & C11 & SHAR & INDIA \\
\hline 68 & Longjiang 1 & DSWLP-A1/LO-93 & Orbiter & CNSA & 20-May-2018 & 21-May-2018 & Long March & IV (4) & XLC-3 & CHINA \\
\hline 69. & Beresheet & & Lander & ISA & 22-Feb-2019 & 04-Apr-2019 & Falcon & Falcon-9-B5 & CC-SLC-40 & ISRAEL \\
\hline 70. & Vikram/Pragyan & & Lander/Rover & ISRO & 22-Jul-2019 & 06-Sep-2019 & GSLV & Mark-III-M1 & SHAR & INDIA \\
\hline
\end{tabular}




\begin{tabular}{|c|c|c|c|c|c|c|c|c|c|c|c|}
\hline \multirow{2}{*}{ S.No } & \multirow{2}{*}{ Spacecraft } & \multirow{2}{*}{ Distinct Name } & \multirow{2}{*}{$\begin{array}{c}\text { No. of } \\
\text { Instruments }\end{array}$} & \multirow{2}{*}{$\begin{array}{l}\text { Spacecraft } \\
\text { Mass }\end{array}$} & \multirow{2}{*}{$\begin{array}{c}\text { Launch } \\
\text { Mass }\end{array}$} & \multirow{2}{*}{$\begin{array}{c}\text { Approx. } \\
\text { Spacecraft } \\
\text { Mass }\end{array}$} & \multirow{2}{*}{$\begin{array}{c}\text { Approx. } \\
\text { Launch } \\
\text { Mass }\end{array}$} & \multirow{2}{*}{$\begin{array}{c}\text { Mission } \\
\text { Duration }\end{array}$} & \multicolumn{2}{|c|}{$\begin{array}{c}\text { Mission Intermission } \\
\text { between }\end{array}$} & \multirow{2}{*}{$\begin{array}{c}\text { Mission } \\
\text { Degradation }\end{array}$} \\
\hline & & & & & & & & & L-Date & D-Date & \\
\hline 01. & Pioneer 0 & Able-1 & 04 & 38 & 38 & 38 & 38 & 0.00089 & 37 & 37 & 01 \\
\hline 02. & Luna E-1 No.1 & & 05 & 156 & 360 & 156 & 360 & 0.00108 & 18 & 20 & 01 \\
\hline 03. & Pioneer 1 & Able-2 & 05 & 34.2 & 38.3 & 36.25 & 38.3 & 1.8 & 01 & 01 & 02 \\
\hline 04. & Luna E-1.No.2 & & 05 & 156 & 360 & 156 & 360 & 0.0012 & 27 & 27 & 01 \\
\hline 05. & Pioneer 2 & Able-3 & 06 & 39.2 & 39.5 & 39.5 & 39.2 & 0.03125 & 26 & 26 & 01 \\
\hline 06. & Luna E-1.No.3 & & 05 & 156 & 360 & 156 & 360 & 0.00284 & 02 & 03 & 01 \\
\hline 07. & Pioneer 3 & & 02 & 5.87 & 5.87 & 5.87 & 5.87 & 1.59 & 27 & 29 & 02 \\
\hline 08. & Mechta / Luna 1 & Luna E-1.No.4 & 05 & 205 & 360 & 181.15 & 282.5 & 2.58 & 60 & 60 & 03 \\
\hline 09. & Pioneer 4 & & 02 & 6.1 & 7.0 & 6.55 & 6.55 & 3.42 & 107 & 104 & 03 \\
\hline 10. & Luna E-1A.No.1 & Luna E-1A.No.5 & 05 & 156 & 390 & 156 & 390 & 0.00186 & 98 & 100 & 01 \\
\hline 11. & Pioneer P-1 & & 10 & 25.3 & 88.4 & 25.3 & 88.4 & - & 63 & 61 & 02 \\
\hline 12. & Pioneer P-3 & Able-IVB & 10 & 168.7 & 169 & 169 & 168.70 & 0.0012 & 141 & 141 & 01 \\
\hline 13. & Luna 1960A & Luna E-3.No.1 & 03 & 279 & 280 & 279.5 & 280 & 0.00844 & 04 & 04 & 01 \\
\hline 14. & Luna 1960B & Luna E-3.No.2 & 03 & 279 & 280 & 279 & 280 & 0.000116 & 159 & 159 & 01 \\
\hline 15. & Pioneer P-30 & Able-VA & 09 & 175.5 & 176 & 175.5 & 175.5 & 0.012 & 81 & 81 & 01 \\
\hline 16. & Pioneer P-31 & Able-VB & 10 & 175.5 & 176 & 176 & 175.5 & 0.000787 & 407 & 505 & 01 \\
\hline 17. & Ranger 3 & $\mathrm{P}-34$ & 04 & 188 & 336 & 259 & 336 & 1.4 & 87 & 83 & 02 \\
\hline 18. & Ranger 4 & P-35 & 04 & 188 & 336 & 259.56 & 336 & 2.67 & 178 & 178 & 03 \\
\hline 19. & Ranger 5 & P-36 & 04 & 203 & 351 & 272.73 & 351 & 0.33 & 78 & 82 & 01 \\
\hline 20. & Sputnik 25 & Luna E-6.No.2 & 02 & 1500 & 2500 & 1500 & 2500 & 0.045 & 30 & 23 & 07 \\
\hline 21. & Luna E-6.No.3 & Luna E-6.No.3 & 02 & 500 & 1420 & 1350 & 1420 & 0.00122 & 58 & 71 & 01 \\
\hline 22. & Luna 4 & Luna E-6.No.4 & 02 & 500 & 1420 & 925 & 1422 & 12 & 223 & 213 & 14 \\
\hline 23. & Kosmos 21 & Cosmos 21 & 09 & 800 & 890 & 800 & 890 & - & 80 & 80 & 03 \\
\hline 24. & Ranger 6 & P-53 & 01 & 172 & 381 & 276.5 & 372.85 & 2.74 & 51 & 48 & 03 \\
\hline 25. & Luna 1964A & Luna E-6.No.6 & 02 & 500 & 1420 & 1420 & 1422 & 0.00566 & 30 & 30 & 01 \\
\hline 26. & Luna E-6.No.5 & Luna E-6.No.5 & 02 & 500 & 1420 & 1420 & 1422 & 0.00394 & 326 & 331 & 01 \\
\hline 27. & Kosmos 60 & Luna E-6.No.9 & 02 & 1600 & 6530 & 1600 & 6530 & - & 29 & 24 & 05 \\
\hline 28. & Luna 1965A & Luna E-6.No.8 & 02 & 1422 & 1470 & 1470 & 1422 & - & 29 & 32 & 01 \\
\hline 29. & Luna 5 & Luna E-6.No.10 & 02 & 300 & 1476 & 888 & 1422.5 & 3 & 30 & 37 & 03 \\
\hline 30. & Luna 6 & Luna E-6.No.7 & 02 & 280 & 1442 & 861 & 1441 & 10 & 118 & 111 & 10 \\
\hline 31. & Luna 7 & Luna E-6.No.11 & 02 & 300 & 1504 & 903 & 1505 & 3 & 60 & 60 & 03 \\
\hline 32. & Luna 8 & Luna E-6.No.12 & 02 & 350 & 1550 & 951 & 1551 & 3 & 88 & 62 & 03 \\
\hline 33. & Kosmos 111 & Luna E-6S.No.204 & 07 & 1580 & 6459 & 1580 & 6459 & 2 & 62 & 58 & 02 \\
\hline 34. & Luna 1966A & & - & 280 & 1600 & 940 & 1600 & - & 40 & 3797 & 01 \\
\hline 35. & Explorer 33 & AIMP-D & 05 & 57 & 94 & 75.2 & 153 & 1876 & 11 & 3615 & 3734 \\
\hline 36. & Lunar Orbiter 1 & Luna Orbiter-A & 03 & 261 & 385 & 323.3 & 382.8 & 78.19 & 30 & 28 & 79.7 \\
\hline 37. & Luna 11 & E-6LF.No.101 & 07 & 1308 & 1640 & 1474 & 1640 & 38 & 200 & 08 & 41.2 \\
\hline 38. & Surveyor 2 & Surveyor-B & 01 & 292 & 1004 & 292 & 999.6 & 2.62 & 97 & 199 & 03 \\
\hline 39. & Kosmos 154 & 7K-L1.No.3P & - & 5017 & 5600 & 5017 & 5600 & 2 & 25 & 98 & 02 \\
\hline
\end{tabular}




\begin{tabular}{|c|c|c|c|c|c|c|c|c|c|c|c|}
\hline \multirow[t]{2}{*}{ S.No } & \multirow[t]{2}{*}{ Spacecraft } & \multirow[t]{2}{*}{ Distinct Name } & \multirow{2}{*}{$\begin{array}{c}\text { No. of } \\
\text { Instruments }\end{array}$} & \multirow{2}{*}{$\begin{array}{l}\text { Spacecraft } \\
\text { Mass }\end{array}$} & \multirow{2}{*}{$\begin{array}{c}\text { Launch } \\
\text { Mass }\end{array}$} & \multirow{2}{*}{$\begin{array}{c}\text { Approx. } \\
\text { Spacecraft } \\
\text { Mass }\end{array}$} & \multirow{2}{*}{$\begin{array}{c}\text { Approx. } \\
\text { Launch } \\
\text { Mass }\end{array}$} & \multirow{2}{*}{$\begin{array}{c}\text { Mission } \\
\text { Duration }\end{array}$} & \multicolumn{2}{|c|}{$\begin{array}{c}\text { Mission Intermission } \\
\text { between }\end{array}$} & \multirow{2}{*}{$\begin{array}{c}\text { Mission } \\
\text { Degradation }\end{array}$} \\
\hline & & & & & & & & & L-Date & D-Date & \\
\hline 40. & Surveyor 4 & Surveyor-D & 03 & 299 & 1042 & 299 & 1040.5 & 2.58 & 75 & 72 & 03 \\
\hline 41. & Zond 1967A & Soyuz 7K-L1.No.4L & - & 5375 & 5390 & 5375 & 5390 & 0.00118 & 56 & 56 & 01 \\
\hline 42. & Zond 1967B & Soyuz 7L-L1.No.5L & - & 5375 & 5390 & 5375 & 5390 & 0.0015 & 101 & 77 & 01 \\
\hline 43. & Luna 1968A & Luna E-6LS.No.112 & - & 5375 & 5600 & 5375 & 5600 & 0.00607 & 24 & 29 & 01 \\
\hline 44. & Zond 4 & 7K-L1.No.6L & - & 2800 & 5680 & 4087.5 & 5410 & - & 77 & 46 & 05 \\
\hline 45. & Zond 1968A & Soyuz 7K-L1.7L & - & 5375 & 5600 & 5375 & 5375 & 0.003125 & 51 & 240 & 01 \\
\hline 46. & Zond 6 & 7K-L1.No.12L & 05 & 2800 & 5680 & 4087.5 & 5527.5 & 7 & 202 & 64 & 07 \\
\hline 47. & Zond 1969A & Soyuz 7K-L1.No.13L & - & 5375 & 5600 & 5375 & 5600 & 0.00579 & 71 & 30 & 01 \\
\hline 48. & Luna 1969A & Luna E-8.No.201 & 07 & 5590 & 5700 & 5590 & 5700 & 0.00059 & 30 & 02 & 01 \\
\hline 49. & Zond L1S-1 & Soyuz 7K-L1S.No.3S & - & 6900 & 6900 & 6900 & 6900 & 0.00081 & 02 & 53 & 01 \\
\hline 50. & Luna 1969B & & - & 5375 & 5700 & 5375 & 5700 & - & 53 & 60 & 01 \\
\hline 51. & Luna 1969C & Luna E-8-5.No.402 & 03 & 5600 & 5600 & 5600 & 5600 & - & 60 & 19 & 01 \\
\hline 52. & Zond L1S-2 & Soyuz 7L-L1S.No.5L & - & 6900 & 6900 & 6900 & 6900 & 0.000118 & 19 & 18 & 01 \\
\hline 53. & Luna 15 & E-8-5.No.401 & 03 & 1360 & 5700 & 3480 & 5700 & 8 & 82 & 68 & 08 \\
\hline 54. & Kosmos 300 & E-8-5.No.403 & 03 & 5600 & 5700 & 5600 & 5700 & - & 29 & 27 & 04 \\
\hline 55. & Kosmos 305 & E-8-5.No.404 & 03 & 5600 & 5700 & 5600 & 5700 & - & 107 & 105 & 02 \\
\hline 56. & Luna 1970A & Luna E-8-5.No.405 & 03 & 5600 & 5700 & 5600 & 5700 & - & 13 & 13 & 01 \\
\hline 57. & Luna 1970B & & 03 & 5600 & 5700 & 5600 & 5700 & - & 493 & 493 & 01 \\
\hline 58. & Soyuz 7K-LOK & 7K-L1E.No.1 & - & 6698 & 9850 & 6698 & 9850 & - & 67 & 76 & 01 \\
\hline 59. & Luna 18 & E-8-5.No.407 & 04 & 1360 & 5700 & 3480 & 5725 & 09 & 448 & 439 & 09 \\
\hline 60. & Soyuz 7K-LOK & 7K-LOK.No.6A & - & 9500 & 9850 & 9500 & 9850 & 0.00124 & 704 & 716 & 01 \\
\hline 61. & Luna 23 & E-8-5M.No.410 & 04 & 1360 & 5800 & 3480 & 4930.5 & 12 & 353 & 348 & 12 \\
\hline 62. & Luna 1975A & Luna E-8-5.No.412 & 04 & 5600 & 5800 & 5600 & 5800 & 07 & 7405 & 6379 & 07 \\
\hline 63. & Hiten & MUSES-A & 02 & 143 & 197 & 146 & 191.2 & 1172 & 700 & 3369 & 1172 \\
\hline 64. & Hagoromo & & 02 & 08 & 08 & 08 & 12 & 1172 & 700 & 3369 & 1172 \\
\hline 65. & HGS-1 & Asiasat-3/PAS-22 & - & 1822 & 3480 & 2611 & 3472.5 & 1644 & 191 & 526 & 1650 \\
\hline 66. & Nozomi & Planet-B & 14 & 262 & 540 & 260 & 538 & 1980 & 3764 & 2089 & 1985 \\
\hline 67. & Chandrayaan 1 & & 14 & 560 & 1380 & 528 & 1380 & 306.8 & 3497 & 3188 & 494 \\
\hline 68. & Longjiang 1 & DSWLP-A1/LO-93 & - & 45 & 45 & 46 & 45 & - & 278 & 318 & - \\
\hline 69. & Beresheet & & 02 & 150 & 585 & 150 & 585 & 47.7 & 150 & 155 & 440 \\
\hline 70. & Vikram/Pragyan & & - & 626 & 1471 & 612.5 & 1471 & - & - & - & 46 \\
\hline
\end{tabular}


Table-6 Mission Objectives of Failed Lunar Missions

\begin{tabular}{|c|c|c|c|c|c|c|c|}
\hline S.No & Spacecraft & Distinct Name & Power & Objective of the Mission & Outcome & Failed Stage & Reason \\
\hline 01. & Pioneer 0 & Able-1 & $60-100$ & Launch Probe into Deep Space & Failed to enter Lunar Orbit & LNH & Launcher Exploded \\
\hline 02. & Luna E-1 No.1 & & - & Intended to Impact Moon & Failed to Orbit & BSB & Launcher Malfunctioned \\
\hline 03. & Pioneer 1 & Able-2 & $60-100$ & Orbit around the Moon & Failed to enter Lunar Orbit & SS & Guidance Failure \\
\hline 04. & Luna E-1.No.2 & & - & Intended to Impact Moon & Failed to Orbit & BSB & Launcher Malfunctioned \\
\hline 05. & Pioneer 2 & Able-3 & $60-100$ & Lunar and Cislunar Probe & Failed to Orbit & BSB & Failed Ignition \\
\hline 06. & Luna E-1.No.3 & & - & Intended to Impact Moon & Failed to Orbit & BSB & Launcher Malfunctioned \\
\hline 07. & Pioneer 3 & & $60-100$ & Flyby around the Moon & Failed to enter Lunar Orbit & BSB & Failed Ignition \\
\hline 08. & Mechta / Luna 1 & Luna E-1.No.4 & - & Intended to Impact Moon & Failed to Orbit & CRC-2|TLI & Failed Ignition \\
\hline 09. & Pioneer 4 & & $60-100$ & Flyby around the Moon & Spacecraft Dead & MCCM & Unable to turn on Photoelectric Sensor \\
\hline 10. & Luna E-1A.No.1 & Luna E-1A.No.5 & - & Intended to Impact Moon & Failed to Orbit & BSB & Launcher Malfunctioned \\
\hline 11. & Pioneer P-1 & & - & Orbit around the Moon & Failed to Launch & LNH & Launcher Exploded \\
\hline 12. & Pioneer P-3 & Able-IVB & - & Orbit around the Moon & Failed to Orbit & BSB & Loss of Telemetry \\
\hline 13. & Luna 1960A & Luna E-3.No.1 & - & Flyby around the Moon & Failed to Orbit & BSB & Launcher Malfunctioned \\
\hline 14. & Luna 1960B & Luna E-3.No.2 & - & Flyby around the Moon & Failed to Orbit & BSB & Launcher Malfunctioned \\
\hline 15. & Pioneer P-30 & Able-VA & - & Place in Lunar Orbit & Failed to Launch & BSB & Launcher Malfunctioned \\
\hline 16. & Pioneer P-31 & Able-VB & - & Place in Lunar Orbit & Failed to Launch & BSB & Loss of Telemetry \\
\hline 17. & Ranger 3 & $\mathrm{P}-34$ & $150 \mathrm{~W}$ & First attempt to land on Moon & Missed the Moon & MCCM & Communication Ceased \\
\hline 18. & Ranger 4 & $\mathrm{P}-35$ & $135 \mathrm{~W}$ & Intended to Impact Moon & Failed before impact & CRC-2|TLI|MCCM & Radio transponder Ceased \\
\hline 19. & Ranger 5 & P-36 & $135 \mathrm{~W}$ & Intended to Impact Moon & Ran out of power & CRC-3 & Radio transponder Ceased \\
\hline 20. & Sputnik 25 & Luna E-6.No.2 & - & Landing Attempt & Never left LEO & BSB & Failed Ignition \\
\hline 21. & Luna E-6.No.3 & Luna E-6.No.3 & - & Soft-landing attempt & Failed to Orbit & BSB|SS & Launch Failure \\
\hline 22. & Luna 4 & Luna E-6.No.4 & - & Soft-landing attempt & Failed to enter Lunar Orbit & MCCM & Spacecraft failed \\
\hline 23. & Kosmos 21 & Cosmos 21 & - & Technology Test Deep Space & Never left LEO & VSS & Loss of Telemetry Signal \\
\hline 24. & Ranger 6 & $\mathrm{P}-53$ & $240 \mathrm{~W}$ & Achieve Lunar Impact Trajectory & Mission Unaccomplished & CRC-7 & Failure of Camera System \\
\hline 25. & Luna 1964A & Luna E-6.No.6 & - & Soft-landing attempt & Failed to Orbit & BSB & Launch Failure \\
\hline 26. & Luna E-6.No.5 & Luna E-6.No.5 & - & Soft-landing attempt & Failed to Orbit & BSB & Launch Failure \\
\hline 27. & Kosmos 60 & Luna E-6.No.9 & - & Soft-landing attempt & Failed to left Orbit & OMB & Failed Ignition \\
\hline 28. & Luna 1965A & Luna E-6.No.8 & - & Soft-landing attempt & Failed to Orbit & BSB & Launch Failure \\
\hline 29. & Luna 5 & Luna E-6.No.10 & - & Soft-landing attempt & Failed to Land & DEI & Retrorocket Failure \\
\hline 30. & Luna 6 & Luna E-6.No.7 & - & Soft-landing attempt & Failed to Land & DEI & Ignition Failure \\
\hline 31. & Luna 7 & Luna E-6.No.11 & - & Soft-landing attempt & Failed to Land & DEI & Premature Retrorocket Shutdown \\
\hline 32. & Luna 8 & Luna E-6.No.12 & - & Soft-landing attempt & Impacted on the Surface & LD & Delayed Retrorocket Ignition \\
\hline 33. & Kosmos 111 & Luna E-6S.No.204 & - & Orbit around Moon & Failed to Orbit & VSS & Launcher Failure \\
\hline 34. & Luna 1966A & & - & Orbit around the Moon & Never reached LEO & LNH & Launcher Failure \\
\hline 35. & Explorer 33 & AIMP-D & - & Intended for Lunar Orbit & Failed to Capture Moon & LOI & Abnormal Velocity \\
\hline 36. & Lunar Orbiter 1 & Luna Orbiter-A & - & Orbit around the Moon & Crashed on the Surface & TOI & Attitude Control System \\
\hline 37. & Luna 11 & E-6LF.No.101 & - & Orbit around the Moon & Spacecraft failed & SSC & Attitude Control System \\
\hline 38. & Surveyor 2 & Surveyor-B & - & Lunar Landing attempt & Crashed on the surface & MCCM & Contact lost \\
\hline 39. & Kosmos 154 & 7K-L1.No.3P & - & Test Spacecraft & Failed to left Orbit & VSS & Ignition Failure \\
\hline 40. & Surveyor 4 & Surveyor-D & - & Lunar Landing attempt & Failed to Land & CRC-6 & Lost Contact before landing \\
\hline 41. & Zond 1967A & Soyuz 7K-L1.No.4L & - & Perform Circumlunar Flyby & Failed to Orbit & BSB & Launch Failure \\
\hline 42. & Zond 1967B & Soyuz 7L-L1.No.5L & - & Perform Circumlunar Flyby & Failed to Orbit & BSB & Launch Failure \\
\hline
\end{tabular}




\begin{tabular}{|c|c|c|c|c|c|c|c|}
\hline S.No & Spacecraft & Distinct Name & Power & Objective of the Mission & Outcome & Failed Stage & Reason \\
\hline 43. & Luna 1968A & Luna E-6LS.No.112 & - & Orbit around the Moon & Failed to Orbit & BSB & Launch Failure \\
\hline 44. & Zond 4 & 7K-L1.No.6L & - & Crewed Flyby Spacecraft Test & Failed to enter Lunar Orbit & LD & Guidance System Failure \\
\hline 45. & Zond 1968A & Soyuz 7K-L1.7L & - & Crewed Lunar Flyby Test & Failed to enter Lunar Orbit & BSB & Launcher Explosion \\
\hline 46. & Zond 6 & 7K-L1.No.12L & - & Crewed Lunar Flyby Test & Crashed on the Earth Surface & VSS & Parachute Failure \\
\hline 47. & Zond 1969A & Soyuz 7K-L1.No.13L & - & Lunar Flyby & Failed to Orbit & $\mathrm{BSB}$ & Launch Failure \\
\hline 48. & Luna 1969A & Luna E-8.No.201 & - & Lunar Landing Attempt & Failed to Orbit & BSB & Launch Failure \\
\hline 49. & Zond L1S-1 & Soyuz 7K-L1S.No.3S & - & NI Launch Test & Failed to Orbit & LNH & Launcher Exploded \\
\hline 50. & Luna 1969B & & - & Sample Return Attempt & Failed to Orbit & LNH & Launch Failure \\
\hline 51. & Luna 1969C & Luna E-8-5.No.402 & - & Sample Return Attempt & Failed to Orbit & LNH & Launch Failure \\
\hline 52. & Zond L1S-2 & Soyuz 7L-L1S.No.5L & - & NI Launch Test & Failed to Orbit & LNH & Launcher Exploded \\
\hline 53. & Luna 15 & E-8-5.No.401 & - & Orbiter and Landing Attempt & Failed to land & EPOI & Failed Ignition \\
\hline 54. & Kosmos 300 & E-8-5.No.403 & - & Lunar Sample Return Attempt & Never Left LEO & OMB & Failed Ignition \\
\hline 55. & Kosmos 305 & E-8-5.No.404 & - & Lunar Sample Return Attempt & Never Left LEO & OMB & Failed Ignition \\
\hline 56. & Luna 1970A & Luna E-8-5.No.405 & & Lunar Sample Return Attempt & Failed to Orbit & BSB & Launch Failure \\
\hline 57. & Luna 1970B & & - & Orbit around the Moon & Failed to Orbit & LNH & Launch Failure \\
\hline 58. & Soyuz 7K-LOK & 7K-L1E.No.1 & - & Crewed Lunar Flyby Test & Failed to Orbit & BSB & Launch Failure \\
\hline 59. & Luna 18 & E-8-5.No.407 & - & Lunar Sample Return Attempt & Failed to Land & LD & Contact Lost \\
\hline 60. & Soyuz 7K-LOK & 7K-LOK.No.6A & - & Crewed Lunar Flyby Test & Failed to Orbit & LNH & Launch Failure \\
\hline 61. & Luna 23 & E-8-5M.No.410 & - & Lunar Sample Return Attempt & Spacecraft Damaged & LL|CRC-6|LGTD & Spacecraft Malfunctioned \\
\hline 62. & Luna 1975A & Luna E-8-5.No.412 & - & Lunar Sample Return Attempt & Failed to Orbit & BSB & Launch Failure \\
\hline 63. & Hiten & MUSES-A & $110 \mathrm{~W}$ & Flyby around Moon & Decayed & CRC-4 & Lack of Fuel \\
\hline 64. & Hagoromo & & $110 \mathrm{~W}$ & Lunar Impact on the Moon & Crashed into the surface & CRC-4 & - \\
\hline 65. & HGS-1 & Asiasat-3/PAS-22 & - & Gravity Assist Probes & - & VSS & - \\
\hline 66. & Nozomi & Planet-B & - & Gravity Assist Probes & - & CRC-2 & Thruster Failure \\
\hline 67. & Chandrayaan 1 & & $700 \mathrm{~W}$ & Orbit around the Moon & Spacecraft Failure & LOI & Thermal Shielding \\
\hline 68. & Longjiang 1 & DSWLP-A1/LO-93 & - & Microsatellite Mission & Never Left LEO & LOI & Spacecraft Malfunctioned \\
\hline 69. & Beresheet & & - & Soft-landing attempt & Crashed into the Surface & LD|LL & Gyroscope Malfunction \\
\hline 70. & Vikram/Pragyan & & $650 \mathrm{~W}$ & Soft-landing attempt & Crashed into the Surface & $\mathrm{LD} \mid \mathrm{LL}$ & Telemetry Lost \\
\hline
\end{tabular}


Table-7 Chronology of Mars Exploration Missions (1960-2020)

\begin{tabular}{|c|c|c|c|c|c|c|c|c|c|}
\hline S.No & Spacecraft & Distinct Name & Type & Instruments & Launch Date & Launcher & Launch Site & Country & Manufacturer \\
\hline 01. & 1M No.1 & Marsnik-1 & Flyby & 03 & 10 Oct 1960 & Molniya 8K78/L1-4 & Baikonur 1/5 & USSR & OKB-1 \\
\hline 02. & $1 \mathrm{M}$ No.2 & Marsnik-2 & Flyby & 03 & 14 Oct 1960 & Molniya 8K78/L1-5 & Baikonur 1/5 & USSR & OKB-1 \\
\hline 03. & 2 MV-4 No.1 & Sputnik-22 & Flyby & 02 & 24 Oct 1962 & Molniya 8K78/T-103-15 & Baikonur 1/5 & USSR & OKB-1 \\
\hline 04. & 2 MV-4 No.4 & Mars-1 & Flyby & 02 & 01 Nov 1962 & Molniya 8K78/T103-16 & Baikonur 1/5 & USSR & OKB-1 \\
\hline 05. & 2 MV-3 No.1 & Sputnik-24 & Lander & - & 04 Nov 1962 & Molniya 8K78/T103-17 & Baikonur 1/5 & USSR & OKB-1 \\
\hline 06. & Mariner 3 & & Flyby & 08 & 05 Nov 1964 & Atlas LV-3 Agena D & CC-LC-13 & USA & NASA/JPL \\
\hline 07. & Mariner 4 & & Flyby & 08 & 28 Nov 1964 & Atlas LV-3 Agena D & CC-LC-12 & USA & NASA/JPL \\
\hline 08. & Zond 2 & 3MV-4A No.2 & Flyby & 08 & 30 Nov 1964 & Molniya 8K78 & Baikonur 1/5 & USSR & OKB-1 \\
\hline 09. & Mariner 6 & & Flyby & 06 & $25 \mathrm{Feb} 1969$ & Atlas SLV-3C Centaur D & CC-LC-36B & USA & NASA/JPL \\
\hline 10. & 2M No.521 & 1969A / M-69 & Orbiter & 09 & 27 Mar 1969 & Proton K/D UR-500 & Baikonur 81/24 & USSR & NPO Lavochkin \\
\hline 11. & Mariner 7 & & Flyby & 06 & 27 Mar 1969 & Atlas SLV-3C Centaur D & CC-LC-36A & USA & NASA/JPL \\
\hline 12. & 2M No.522 & 1969B / M-69 & Orbiter & 09 & 02 Apr 1969 & Proton K/D UR-500 & Baikonur 81/23 & USSR & NPO Lavochkin \\
\hline 13. & Mariner 8 & & Orbiter & 06 & 09 May 1971 & Atlas SLV-3C Centaur D & Baikonur 81/24 & USA & NASA/JPL \\
\hline 14. & Kosmos 419 & & Orbiter & 11 & 10 May 1971 & Proton K/D UR-500 & Baikonur 81/24 & USSR & GSMZ Lavochkin \\
\hline 15. & Mars 2 (M-71) & 4M No.171 & Orbiter & 11 & 19 May 1971 & Proton K/D UR-500 & Baikonur 81/24 & USSR & OKB-1 \\
\hline 16. & Mars 2 (M-71) & SA 4M No.171 & Lander & 08 & 19 May 1971 & Proton K/D UR-500 & Baikonur 81/24 & USSR & OKB-1 \\
\hline 17. & Prop-M Rover & M-71 - Rover & Rover & 02 & 19 May 1971 & Proton K/D UR-500 & Baikonur 81/24 & USSR & OKB-1 \\
\hline 18. & Mars 3 (M-71) & 4M No.172 & Orbiter & 10 & 28 May 1971 & Proton K/D UR-500 & Baikonur 81/24 & USSR & GSMZ Lavochkin \\
\hline 19. & Mars 3 (M-71) & SA 4M No.172 & Lander & 08 & 28 May 1971 & Proton K/D UR-500 & Baikonur 81/24 & USSR & GSMZ Lavochkin \\
\hline 20. & Prop-M Rover & M-71 - Rover & Rover & 02 & 28 May 1971 & Proton K/D UR-500 & Baikonur 81/24 & USSR & GSMZ Lavochkin \\
\hline 21 & Mariner 9 & & Orbiter & 06 & 30 May 1971 & Atlas SLV-3C Centaur D & CC-LC-36B & USA & NASA/JPL \\
\hline 22. & Mars 4 & 3MS No.52S & Orbiter & 15 & $21 \mathrm{Jul} 1973$ & Proton K/D UR-500 & Baikonur 81/23 & USSR & GSMZ Lavochkin \\
\hline 23. & Mars 5 & 3MS No.53S & Orbiter & 15 & 25 Jul 1973 & Proton K/D UR-500 & Baikonur 81/24 & USSR & GSMZ Lavochkin \\
\hline 24. & Mars 6 & 3MP No.50P & Flyby & 05 & 05 Aug 1973 & Proton K/D UR-500 & Baikonur 81/23 & USSR & GSMZ Lavochkin \\
\hline 25. & Mars 6 & 3MP No.50P & Lander & 06 & 05 Aug 1973 & Proton K/D UR-500 & Baikonur 81/23 & USSR & GSMZ Lavochkin \\
\hline 26. & Mars 7 & 3 MP No.51P & Flyby & 06 & 09 Aug 1973 & Proton K/D UR-500 & Baikonur 81/24 & USSR & GSMZ Lavochkin \\
\hline 27. & Mars 7 & 3 MP No.51P & Lander & 06 & 09 Aug 1973 & Proton K/D UR-500 & Baikonur 81/24 & USSR & GSMZ Lavochkin \\
\hline 28. & Viking 1 & & Orbiter & 03 & 20 Aug 1975 & Titan IIIE Centaur D1T & CC-LC-41 & USA & NASA/JPL \\
\hline 29. & Viking 1 & & Lander & 09 & 20 Aug 1975 & Titan IIIE Centaur D1T & CC-LC-41 & USA & Martin Marietta \\
\hline 30. & Viking 2 & & Orbiter & 03 & 09 Sep 1975 & Titan IIIE Centaur D1T & CC-LC-41 & USA & NASA/JPL \\
\hline 31. & Viking 2 & & Lander & 09 & 09 Sep 1975 & Titan IIIE Centaur D1T & CC-LC-41 & USA & Martin Marietta \\
\hline 32. & Phobos 1 & 1F No.101 & Orbiter & 22 & 07 Jul 1988 & Proton K/D UR-500 & Baikonur 200/40 & USSR & NPO Lavochkin \\
\hline 33. & Phobos 1 & 1F No.101 & Lander & - & 07 Jul 1988 & Proton K/D UR-500 & Baikonur 200/40 & USSR & NPO Lavochkin \\
\hline 34. & Phobos 2 & 1F No.102 & Orbiter & 22 & 12 Jul 1988 & Proton K/D UR-500 & Baikonur 200/40 & USSR & NPO Lavochkin \\
\hline 35. & Phobos 2 & 1F No.102 & Lander & - & 12 Jul 1988 & Proton K/D UR-500 & Baikonur 200/40 & USSR & NPO Lavochkin \\
\hline 36. & Mars Observer & & Orbiter & 08 & 25 Sep 1992 & Titan III & CC-LC-40 & USA & NASA/JPL \\
\hline 37. & Mars GS & & Orbiter & 06 & 07 Nov 1996 & Delta-II 7925 & CC-LC-17A & USA & NASA/JPL \\
\hline 38. & Mars 96 & M1 No.520 & Orbiter & 22 & 16 Nov 1996 & Proton K/D-2 UR-500 & Baikonur 200/39 & USSR & NPO Lavochkin \\
\hline 39. & Mars 96 & M1 No.520 & Lander & 06 & 16 Nov 1996 & Proton K/D-2 UR-500 & Baikonur 200/39 & USSR & NPO Lavochkin \\
\hline 40. & Mars 96 & M1 No.520 & Penetrator & 10 & 16 Nov 1996 & Proton K/D-2-UR-500 & Baikonur 200/39 & USSR & NPO Lavochkin \\
\hline 41. & Mars Pathfinder & & Lander & 02 & 04 Dec 1996 & Delta-II 7925 & CC-SLC-17 & USA & NASA/JPL \\
\hline 42. & Sojourner & & Rover & 06 & 04 Dec 1996 & Delta-II 7925 & CC-SLC-17 & USA & NASA/JPL \\
\hline
\end{tabular}




\begin{tabular}{|c|c|c|c|c|c|c|c|c|c|}
\hline S.No & Spacecraft & Distinct Name & Type & Instruments & Launch Date & Launcher & Launch Site & Country & Manufacturer \\
\hline 43. & Nozomi & Planet-B & Orbiter & 14 & 03 Jul 1998 & MV & USC & Japan & JAXA \\
\hline 44. & MCO & MS-98 & Orbiter & 03 & 11 Dec 1998 & Delta II 7425 & CC-LC-17A & US & LMSS \\
\hline 45. & MPL & MS-98 & Lander & 03 & 03 Jan 1999 & Delta II 7425 & CC-LC-17A & US & Martin Marietta \\
\hline 46. & Deep Space 2 & & Penetrators & - & 03 Jan 1999 & Delta II 7425 & CC-LC-17A & US & Martin Marietta \\
\hline 47. & Mars Odyssey & & Orbiter & 03 & 07 Apr 2001 & Delta II 7925 & CC-LC-17A & US & LMSS \\
\hline 48. & Mars Express & & Orbiter & 09 & 02 Jun 2003 & Soyuz-FG/Fregat & Baikonur 31/6 & Europe & ESA \\
\hline 49. & Beagle 2 & & Lander & 04 & 02 Jun 2003 & Soyuz-FG/Fregat & Baikonur 31/6 & Europe & ESA \\
\hline 50. & Spirit & MER-A & Rover & 09 & 10 Jun 2003 & Delta II 7925 & CC-LC-17A & US & NASA/JPL \\
\hline 51. & Opportunity & MER-B & Rover & 09 & $08 \mathrm{Jul} 2003$ & Delta II 7925-H & CC-LC-17A & US & NASA/JPL \\
\hline 52. & Rosetta & & Gravity Assist & 11 & 02 Mar 2004 & Ariane 5G+ & Kourou ELA-3 & Europe & Astrium/ESA \\
\hline 53. & MRO & & Orbiter & 06 & 12 Aug 2005 & Atlas V 401 & CC-SLC-41 & US & LMSS \\
\hline 54. & Phoenix & & Lander & 11 & 04 Aug 2007 & Delta II 7925 & CC-SLC-17A & US & LMSS \\
\hline 55. & Dawn & & Gravity Assist & 03 & 27 Sep 2007 & Delta II 7925-H & CC-SLC-17B & US & NASA/JPL \\
\hline 56. & Fobos-Grunt & & Orbiter & 16 & 08 Nov 2011 & Zenit-2M/2FG & Baikonur 45/1 & USSR & NPO Lavochkin \\
\hline 57. & Fobos-Grunt & & Sample Return & - & 08 Nov 2011 & Zenit-2M/2FG & Baikonur 45/1 & USSR & NPO Lavochkin \\
\hline 58. & Yinguo-1 & & Orbiter & 04 & 08 Nov 2011 & Zenit-2M/2FG & Baikonur 45/1 & China & CNSA \\
\hline 59. & Curiosity & MSL & Rover & 12 & 26 Nov 2011 & Atlas V 541 & CC-LC-41 & US & NASA/JPL \\
\hline 60. & Mangalyaan & MOM & Orbiter & 05 & 05 Nov 2013 & PSLV-XL & SHAR & India & ISRO \\
\hline 61. & MAVEN & & Orbiter & 08 & 18 Nov 2013 & Atlas V 401 & CC-SLC-41 & US & NASA/GSFC \\
\hline 62. & ExoMars TGO & & Orbiter & 04 & 14 Mar 2016 & Proton-M/Briz-M & Baikonur 200/39 & Europe & TAS/ESA \\
\hline 63. & Schiaparelli EDM & & Lander & - & 14 Mar 2016 & Proton-M/Briz-M & Baikonur 200/39 & Europe & TAS/ESA \\
\hline 64. & InSight & & Lander & 04 & 05 May 2018 & Atlas V 401 & Vandenberg SLC-3E & US & LMSS \\
\hline 65. & MarCo-A & & Flyby & - & 05 May 2018 & Atlas V 401 & Vandenberg SLC-3E & US & NASA/JPL \\
\hline 66. & MarCo-B & & Flyby & - & 05 May 2018 & Atlas V 401 & Vandenberg SLC-3E & US & NASA/JPL \\
\hline 65. & Tianwen-1 & Huoxing-1 & Orbiter & - & 23-Jul-2020 & Long March 5 & Wenchang-LC-101 & CHINA & CNSA \\
\hline 66. & Tianwen-1 & & Lander & - & 23-Jul-2020 & Long March 5 & Wenchang-LC-101 & CHINA & CNSA \\
\hline 67. & Tianwen-1 & & Rover & - & 23-Jul-2020 & Long March 5 & Wenchang-LC-101 & CHINA & CNSA \\
\hline 68. & Perseverance Rover & Mars 2020 & Rover & - & 30-Jul-2020 & Atlas V 541 (AV-088) & CC-SLC-41 & US & NASA/JPL \\
\hline 69 & Ingenuity & & Helicopter & - & 30-Jul-2020 & Atlas V 541 (AV-088) & CC-SLC-41 & US & NASA/JPL \\
\hline
\end{tabular}


Table-8 Spacecraft Parameters of Mars Missions

\begin{tabular}{|c|c|c|c|c|c|c|c|c|c|c|c|}
\hline \multirow{2}{*}{ S.No } & \multirow{2}{*}{ Spacecraft Name } & \multirow{2}{*}{ : } & \multirow{2}{*}{ Probe Type } & \multirow{2}{*}{ Launch Date } & \multirow{2}{*}{ Decay Date } & \multirow{2}{*}{$\begin{array}{c}\text { Spacecraft } \\
\text { Mass } \\
\text { (kg) }\end{array}$} & \multirow{2}{*}{$\begin{array}{c}\text { Launch } \\
\text { Mass } \\
\text { (kg) }\end{array}$} & \multirow{2}{*}{$\begin{array}{c}\text { Mission } \\
\text { Duration } \\
\text { (Days) }\end{array}$} & \multicolumn{2}{|c|}{ Mission Intermission between } & \multirow{2}{*}{$\begin{array}{c}\text { Mission } \\
\text { Degradation } \\
\text { (Days) }\end{array}$} \\
\hline & & & & & & & & & $\begin{array}{l}\text { Launch } \\
\text { Date (Days) }\end{array}$ & $\begin{array}{l}\text { Decay } \\
\text { Date (Davs) }\end{array}$ & \\
\hline 01. & \begin{tabular}{|l|} 
1M No.1 \\
\end{tabular} & \multirow{12}{*}{ 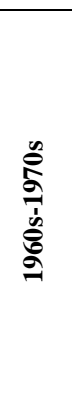 } & Flyby & 10 Oct 1960 & 10-Oct-1960 & 480 & 650 & 0.0036 & 04 & 04 & 01 \\
\hline 02. & $1 \mathrm{M}$ No. 2 & & Flyby & 14 Oct 1960 & 14-Oct-1960 & 480 & 650 & 0.0034 & 740 & 865 & 01 \\
\hline 03. & 2 MV-4 No.1 & & Flyby & 24 Oct 1962 & 26-Feb-1963 & 893 & 900 & 0.0034 & 08 & 113 & 125 \\
\hline 04. & 2 MV-4 No.2 & & Flyby & \begin{tabular}{|l|}
01 Nov 1962 \\
\end{tabular} & 19-Jun-1963 & 893 & 900 & 140 & 03 & 657 & 230 \\
\hline 05. & 2 MV-3 No.1 & & Lander & 04 Nov 1962 & 19-Jan-1963 & \begin{tabular}{|l|}
890 \\
\end{tabular} & 900 & 0.0030 & 732 & 657 & 227 \\
\hline 06. & \begin{tabular}{|l|} 
Mariner 3 \\
\end{tabular} & & Flyby & 05 Nov 1964 & 06-Nov-1964 & 260 & 397 & 0.36 & 23 & 1140 & 01 \\
\hline 07. & Mariner 4 & & Flyby & 28 Nov 1964 & 21-Dec-1967 & 244 & 260 & 1118 & 02 & 1098 & 1118 \\
\hline 08. & Zond 2 & & Flyby & 30 Nov 1964 & 18-Dec-1964 & \begin{tabular}{|r|}
890 \\
\end{tabular} & 996 & 249 & 1548 & 2196 & 249 \\
\hline 09. & Mariner 6 & & Flyby & 25 Feb 1969 & 23-Dec-1970 & \begin{tabular}{|l|}
381 \\
\end{tabular} & 411.8 & 666 & 30 & 636 & 666 \\
\hline 10. & $2 \mathrm{M}$ No.521 & & Orbiter & 27 Mar 1969 & 27-Mar-1969 & 3800 & 4850 & 0.0050 & 30 & 641 & 01 \\
\hline 11. & \begin{tabular}{|l|} 
Mariner 7 \\
\end{tabular} & & Flyby & 27 Mar 1969 & 28-Dec-1970 & 381 & 411.8 & 640 & 06 & 635 & 641 \\
\hline 12. & $2 \mathrm{M} \mathrm{No.522}$ & & Orbiter & 02 Apr 1969 & 02-Apr-1969 & \begin{tabular}{|l|}
3800 \\
\end{tabular} & 4850 & 0.00048 & 767 & \begin{tabular}{|l|}
767 \\
\end{tabular} & 01 \\
\hline 13. & \begin{tabular}{|l|} 
Mariner 8 \\
\end{tabular} & \multirow{19}{*}{ 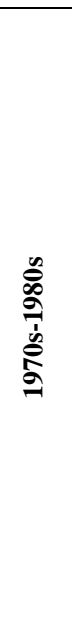 } & Orbiter & 09 May 1971 & 09-May-1971 & \begin{tabular}{|l|}
558.8 \\
\end{tabular} & 997.9 & 0.0032 & 01 & 03 & 01 \\
\hline 14. & Kosmos 419 & & Orbiter & 10 May 1971 & 12-May-1971 & \begin{tabular}{|l|}
4549 \\
\end{tabular} & - & 0.0625 & 09 & 468 & 02 \\
\hline 15. & \begin{tabular}{|l} 
Mars 2(M-71) \\
\end{tabular} & & Orbiter & 19 May 1971 & 22-Aug-1972 & \begin{tabular}{|l|}
2265 \\
\end{tabular} & 3440 & 461 & 09 & \begin{tabular}{|l|l|}
269 \\
\end{tabular} & 461 \\
\hline 16. & Mars 2(M-71) & & Lander & 19 May 1971 & 27-Nov-1971 & 358 & 1210 & 192 & 09 & 269 & 192 \\
\hline 17. & Prop-M Rover & & Rover & 19 May 1971 & 27-Nov-1971 & 4.5 & - & - & 09 & 269 & 192 \\
\hline 18. & Mars $3(\mathrm{M}-71)$ & & Orbiter & 28 May 1971 & 22-Aug-1972 & 2265 & 3440 & 452 & 02 & 264 & 452 \\
\hline 19. & $\begin{array}{l}\text { Mars } 3 \text { (M-71) } \\
\end{array}$ & & Lander & 28 May 1971 & 02-Dec-1971 & \begin{tabular}{|l|}
358 \\
\end{tabular} & 1210 & 188 & 02 & 330 & 188 \\
\hline 20. & Prop-M Rover & & Rover & 28 May 1971 & 02-Dec-1971 & \begin{tabular}{|l|l|}
4.5 \\
\end{tabular} & - & - & 02 & 330 & 188 \\
\hline 21 & Mariner 9 & & Orbiter & 30 May 1971 & 27-Oct-1972 & 558.8 & 997.9 & 514 & 783 & \begin{tabular}{|l|l}
471 \\
470
\end{tabular} & 516 \\
\hline 22. & Mars 4 & & Orbiter & 21 Jul 1973 & 10-Feb-1974 & \begin{tabular}{|l|}
2265 \\
\end{tabular} & 3440 & 195 & \begin{tabular}{|l|l}
04 \\
\end{tabular} & 18 & 204 \\
\hline 23. & Mars 5 & & Orbiter & \begin{tabular}{|l|}
$25 \mathrm{Jul} 1973$ \\
\end{tabular} & 28-Feb-1974 & 2265 & 3440 & 218 & 11 & 12 & 218 \\
\hline 24. & Mars 6 & & Flyby & 05 Aug 1973 & 12-Mar-1974 & 1900 & 3260 & 219 & 04 & 13 & 219 \\
\hline 25. & Mars 6 & & Lander & 05 Aug 1973 & 12-Mar-1974 & 635 & 3260 & 219 & 04 & 13 & 219 \\
\hline 26. & \begin{tabular}{|l|} 
Mars 7 \\
\end{tabular} & & Flyby & 09 Aug 1973 & 25-Mar-1974 & \begin{tabular}{|l|}
1900 \\
\end{tabular} & 3260 & 214 & 741 & 16 & 228 \\
\hline 27. & Mars 7 & & Lander & 09 Aug 1973 & 09-Mar-1974 & \begin{tabular}{|l|l|}
635 \\
\end{tabular} & 3260 & 214 & 741 & 3169 & 212 \\
\hline 28. & \begin{tabular}{|l|} 
Viking 1 \\
\end{tabular} & & Orbiter & 20 Aug 1975 & 17-Sep-1980 & \begin{tabular}{|l|}
883 \\
\end{tabular} & 3530 & 1824 & 20 & 2305 & 1846 \\
\hline 29. & Viking 1 & & Lander & 20 Aug 1975 & 11-Nov-1982 & 572 & 3530 & 2040 & 20 & 1362 & 2640 \\
\hline 30. & \begin{tabular}{|l|} 
Viking 2 \\
\end{tabular} & & Orbiter & 09 Sep 1975 & 25-Jul-1973 & 883 & 3530 & 1050 & 4685 & 1317 & 1050 \\
\hline 31. & \begin{tabular}{|l|} 
Viking 2 \\
\end{tabular} & & Lander & 09 Sep 1975 & 12-Apr-1980 & \begin{tabular}{|l|}
572 \\
\end{tabular} & 3530 & 1677 & 4685 & 4444 & 1677 \\
\hline 32. & \begin{tabular}{|l|} 
Phobos 1 \\
\end{tabular} & \multirow{4}{*}{ 容 } & Orbiter & \begin{tabular}{|l|} 
07 Jul 1988 \\
\end{tabular} & 03-Nov-1988 & 2420 & 6220 & 52 & 05 & 144 & 56 \\
\hline 33. & \begin{tabular}{|l|} 
Phobos 1 \\
\end{tabular} & & Lander & \begin{tabular}{|l|} 
07 Jul 1988 \\
\end{tabular} & 03-Nov-1988 & \begin{tabular}{|l|}
570 \\
\end{tabular} & 3800 & 52 & 05 & 144 & 56 \\
\hline 34. & $\begin{array}{l}\text { Phobos } 2 \\
\end{array}$ & & Orbiter & 12 Jul 1988 & 27-Mar-1989 & \begin{tabular}{|l|}
2420 \\
\end{tabular} & 6220 & 258 & 1536 & 19 & 258 \\
\hline 35. & \begin{tabular}{|l|} 
Phobos 2 \\
\end{tabular} & & Lander & 12 Jul 1988 & 15-Apr-1989 & 570 & 3800 & 258 & 1536 & 1589 & 277 \\
\hline 36. & Mars Observer & \multirow{11}{*}{ 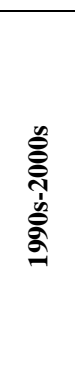 } & Orbiter & 25 Sep 1992 & 21-Aug-1993 & 1018 & 2565 & 330 & 1504 & \begin{tabular}{|l|}
4821 \\
\end{tabular} & 340 \\
\hline 37. & \begin{tabular}{|l|} 
Mars GS \\
\end{tabular} & & Orbiter & 07 Nov 1996 & 02-Nov-2006 & 1030 & 1062 & 3647 & 09 & 3636 & 3700 \\
\hline 38. & \begin{tabular}{|l|} 
Mars 96 \\
\end{tabular} & & Orbiter & 16 Nov 1996 & 18-Nov-1996 & 3780 & 6700 & 0.0062 & 18 & 313 & 02 \\
\hline 39. & Mars 96 & & Lander & 16 Nov 1996 & 18-Nov-1996 & 75 & - & - & 18 & 313 & 02 \\
\hline 40. & Mars 96 & & Penetrator & 16 Nov 1996 & 18-Nov-1996 & 120 & - & - & 18 & 313 & 02 \\
\hline 41. & Mars Pathfinder & & Lander & 04 Dec 1996 & 27-Sep-1997 & \begin{tabular}{|l|}
210 \\
\end{tabular} & 295 & 297 & 576 & 78 & 297 \\
\hline 42. & Sojourner & & Rover & 04 Dec 1996 & 11-Jul-1997 & \begin{tabular}{|l|}
11 \\
\end{tabular} & 11 & 219 & 576 & 2342 & 219 \\
\hline 43. & Nozomi & & Orbiter & 03 Jul 1998 & 09-Dec-2003 & \begin{tabular}{|l|}
258 \\
\end{tabular} & 356 & 1983 & 161 & 1538 & 1985 \\
\hline 44. & $\mathrm{MCO}$ & & Orbiter & 11 Dec 1998 & 23-Sep-1999 & 338 & 638 & 286 & 23 & 71 & 288 \\
\hline 45. & MPL & & Lander & 03 Jan 1999 & 03-Dec-1999 & \begin{tabular}{|l|}
290 \\
\end{tabular} & 583 & 334 & 825 & 1483 & 379 \\
\hline 46. & Deep Space 2 & & Penetrators & 03 Jan 1999 & 03-Dec-1999 & \begin{tabular}{|l|}
2.4 \\
\end{tabular} & - & 334 & 825 & \begin{tabular}{|l|l|}
1483 \\
\end{tabular} & 379 \\
\hline
\end{tabular}




\begin{tabular}{|c|c|c|c|c|c|c|c|c|c|c|c|}
\hline \multirow{2}{*}{ S.No } & \multirow{2}{*}{ Spacecraft Name } & \multirow{2}{*}{ 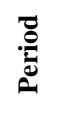 } & \multirow{2}{*}{ Type } & \multirow{2}{*}{ Launch Date } & \multirow{2}{*}{ Decay Date } & \multirow{2}{*}{$\begin{array}{l}\text { Spacecraft } \\
\text { Mass } \\
(\text { kg) }\end{array}$} & \multirow{2}{*}{$\begin{array}{c}\text { Launch } \\
\text { Mass } \\
\text { (kg) }\end{array}$} & \multirow{2}{*}{$\begin{array}{c}\text { Mission } \\
\text { Duration } \\
\text { (Days) }\end{array}$} & \multicolumn{2}{|c|}{ Mission Intermission between } & \multirow{2}{*}{$\begin{array}{c}\text { Mission } \\
\text { Degradation } \\
\text { (Days) }\end{array}$} \\
\hline & & & & & & & & & $\begin{array}{l}\text { Launch } \\
\text { Date (Days) }\end{array}$ & $\begin{array}{l}\text { Decay } \\
\text { Date (Days) }\end{array}$ & \\
\hline 47. & Mars Odyssey & \multirow{6}{*}{ 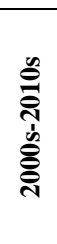 } & Orbiter & 07 Apr 2001 & Operational & 376 & 758 & 6964 & 786 & - & - \\
\hline 49. & Beagle 2 & & Lander & 02 Jun 2003 & 25-Dec-2003 & 09 & 33.2 & 206 & 08 & 2279 & 244 \\
\hline 50. & Spirit & & Rover & 10 Jun 2003 & 22-Mar-2010 & 174 & 1063 & 2477 & 28 & 3002 & 2906 \\
\hline 51. & Opportunity & & Rover & $08 \mathrm{Jul} 2003$ & 10-Jun-2018 & 185 & 1063 & 5452 & 766 & 3507 & 5700 \\
\hline 52. & MRO & & Orbiter & 12 Aug 2005 & Operational & 984 & 2180 & 5376 & 722 & - & - \\
\hline 53. & Phoenix & & Lander & 04 Aug 2007 & 02-Nov-2008 & 350 & 680 & 456 & 1557 & 456 & 1024 \\
\hline 55. & Fobos-Grunt & \multirow{13}{*}{ 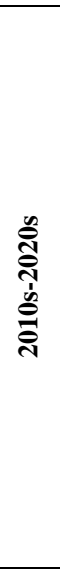 } & Sample Return & 08 Nov 2011 & 15-Jan-2012 & 106 & 296 & - & 18 & 68 & 68 \\
\hline 56. & Yinguo-1 & & Orbiter & 08 Nov 2011 & 15-Jan-2012 & 115 & - & - & 18 & 68 & 68 \\
\hline 57. & Curiosity & & Rover & 26 Nov 2011 & Operational & 899 & - & 3079 & 710 & - & - \\
\hline 58. & Mangalyaan & & Orbiter & 05 Nov 2013 & Operational & 482 & 1337 & 2369 & 13 & - & - \\
\hline 59. & MAVEN & & Orbiter & 18 Nov 2013 & Operational & 809 & 2454 & 2356 & 847 & - & - \\
\hline 60. & ExoMars TGO & & Orbiter & 14 Mar 2016 & Operational & 690.8 & 4332 & 1509 & 71 & - & - \\
\hline 61. & Schiaparelli EDM & & Lander & 14 Mar 2016 & 19-Oct-2016 & 280 & 600 & 219 & 71 & 219 & 219 \\
\hline 62. & InSight & & Lander & 05 May 2018 & Operational & 358 & 694 & 727 & 810 & - & - \\
\hline 63. & MarCo-A & & Flyby & 05 May 2018 & 04-Jan-2019 & 13.5 & 13.5 & 244 & 810 & 244 & 638 \\
\hline 66. & Tianwen-1 & & Lander & 23-Jul-2020 & In Trajectory Phase & 1585 & 5000 & - & 07 & - & - \\
\hline 67. & Tianwen-1 & & Rover & 23-Jul-2020 & In Trajectory Phase & 240 & 5000 & - & 07 & - & - \\
\hline 68. & Perseverance Rover & & Rover & 30-Jul-2020 & In Trajectory Phase & 1050 & - & - & - & - & - \\
\hline 69 & Ingenuity & & Helicopter & 30-Jul-2020 & In Trajectory Phase & 1.8 & - & - & - & - & - \\
\hline
\end{tabular}




\section{Table-9 Spaceflight Sequence Chart - Failed Lunar Missions}

\begin{tabular}{|c|c|c|c|c|c|c|c|c|c|c|c|c|c|c|c|c|c|c|c|c|c|c|c|c|c|c|c|c|}
\hline S.No & Spacecraft & Distinct Name & LNH & BSB & ss & CRC-1 & EPOI & VSs & OMB & CRC-2 & $\mathrm{TLI}$ & MCCM & LA & CRC-3 & OAB & LOI & LMS & CRC-4 & EITOI & TOI & ssc & DEI & CRC-5 & LD & $\underline{L}$ & CRC-6 & LGTD & CRC-7 \\
\hline 01. & Pioneer 0 & Able-1 & $\otimes$ & & & & & & & & & & & & & & & & & & & & & & & & & \\
\hline 02. & Luna E-1 No.1 & & $\bullet$ & $\otimes$ & & & & & & & & & & & & & & & & & & & & & & & & \\
\hline 03. & Pioneer 1 & Able-2 & $\bullet$ & $\bullet$ & $\otimes$ & & & & & & & & & & & & & & & & & & & & & & & \\
\hline 04. & Luna E-1.No.2 & & $\bullet$ & $\otimes$ & & & & & & & & & & & & & & & & & & & & & & & & \\
\hline 05. & Pioneer 2 & Able-3 & $\bullet$ & $\otimes$ & & & & & & & & & & & & & & & & & & & & & & & & \\
\hline 06. & Luna E-1.No.3 & & $\bullet$ & $\otimes$ & & & & & & & & & & & & & & & & & & & & & & & & \\
\hline 07. & Pioneer 3 & & $\bullet$ & $\otimes$ & & & & & & & & & & & & & & & & & & & & & & & & \\
\hline 08. & Mechta / Luna 1 & Luna E-1.No.4 & $\bullet$ & $\bullet$ & $\bullet$ & $\bullet$ & $\bullet$ & - & $\bullet$ & $\otimes$ & $\otimes$ & & & & & & & & & & & & & & & & & \\
\hline 09. & Pionecr 4 & & $\bullet$ & - & - & - & • & - & - & $\bullet$ & - & $\otimes$ & & & & & & & & & & & & & & & & \\
\hline 10. & Luna E-1A.No.1 & Luna E-1A.No.5 & $\bullet$ & $\otimes$ & & & & & & & & & & & & & & & & & & & & & & & & \\
\hline 11. & Pioneer P-1 & & $\otimes$ & & & & & & & & & & & & & & & & & & & & & & & & & \\
\hline 12. & Pioneer P-3 & Able-IVB & $\bullet$ & $\otimes$ & & & & & & & & & & & & & & & & & & & & & & & & \\
\hline 13. & Luna $1960 \mathrm{~A}$ & Luna E-3.No.1 & $\bullet$ & $\otimes$ & & & & & & & & & & & & & & & & & & & & & & & & \\
\hline 14. & Luna 1960B & Luna E-3.No.2 & $\bullet$ & $\otimes$ & & & & & & & & & & & & & & & & & & & & & & & & \\
\hline 15. & Pioneer P-30 & Able-VA & $\bullet$ & $\otimes$ & & & & & & & & & & & & & & & & & & & & & & & & \\
\hline 16. & Pioneer P-31 & Able-VB & $\bullet$ & $\otimes$ & & & & & & & & & & & & & & & & & & & & & & & & \\
\hline 17. & Ranger 3 & P-34 & $\bullet$ & $\bullet$ & $\bullet$ & $\bullet$ & $\bullet$ & $\cdot$ & $\bullet$ & $\bullet$ & $\bullet$ & $\otimes$ & & & & & & & & & & & & & & & & \\
\hline 18. & Ranger 4 & P-35 & $\bullet$ & $\bullet$ & $\bullet$ & $\bullet$ & $\cdot$ & $\cdot$ & $\bullet$ & $\otimes$ & $\otimes$ & $\otimes$ & & & & & & & & & & & & & & & & \\
\hline 19. & Ranger 5 & P-36 & $\bullet$ & $\bullet$ & $\bullet$ & $\bullet$ & $\bullet$ & $\bullet$ & $\bullet$ & $\bullet$ & $\bullet$ & $\bullet$ & $\bullet$ & $\otimes$ & & & & & & & & & & & & & & \\
\hline 20. & Sputnik 25 & Luna E-6.No.2 & $\bullet$ & $\otimes$ & & & & & & & & & & & & & & & & & & & & & & & & \\
\hline 21. & Luna E-6.No.3 & Luna E-6.No.3 & $\bullet$ & $\otimes$ & $\otimes$ & & & & & & & & & & & & & & & & & & & & & & & \\
\hline 22. & Luna 4 & Luna E-6.No.4 & $\bullet$ & $\bullet$ & $\bullet$ & $\bullet$ & $\bullet$ & $\bullet$ & $\bullet$ & $\bullet$ & $\bullet$ & $\otimes$ & & & & & & & & & & & & & & & & \\
\hline 23. & Kosmos 21 & \begin{tabular}{|l|} 
Cosmos 21 \\
\end{tabular} & $\bullet$ & $\bullet$ & $\bullet$ & $\bullet$ & $\bullet$ & $\otimes$ & & & & & & & & & & & & & & & & & & & & \\
\hline 24. & Ranger 6 & P-53 & $\bullet$ & $\bullet$ & $\bullet$ & $\bullet$ & $\cdot$ & $\bullet$ & $\bullet$ & $\bullet$ & $\bullet$ & $\bullet$ & $\bullet$ & $\bullet$ & $\bullet$ & $\bullet$ & $\bullet$ & $\bullet$ & $\cdot$ & $\bullet$ & $\bullet$ & $\bullet$ & $\bullet$ & $\bullet$ & $\bullet$ & $\bullet$ & $\cdot$ & $\otimes$ \\
\hline 25. & Luna 1964A & Luna E-6.No.6 & $\bullet$ & - & $\otimes$ & & & & & & & & & & & & & & & & & & & & & & & \\
\hline 26. & Luna E-6.No.5 & Luna E-6.No.5 & $\bullet$ & - & $\otimes$ & & & & & & & & & & & & & & & & & & & & & & & \\
\hline 27. & Kosmos 60 & Luna E-6.No.9 & $\bullet$ & $\bullet$ & $\bullet$ & $\bullet$ & $\bullet$ & $\bullet$ & $\otimes$ & & & & & & & & & & & & & & & & & & & \\
\hline 28. & Luna $1965 \mathrm{~A}$ & Luna E-6.No.8 & $\bullet$ & - & $\otimes$ & & & & & & & & & & & & & & & & & & & & & & & \\
\hline 29. & Luna 5 & Luna E-6.No.10 & $\bullet$ & $\bullet$ & $\bullet$ & $\bullet$ & $\bullet$ & $\bullet$ & $\bullet$ & $\bullet$ & $\bullet$ & $\bullet$ & $\bullet$ & $\bullet$ & $\bullet$ & $\bullet$ & $\bullet$ & $\bullet$ & $\bullet$ & $\bullet$ & $\bullet$ & $\otimes$ & & & & & & \\
\hline 30. & Luna 6 & Luna E-6.No.7 & $\bullet$ & $\cdot$ & $\bullet$ & $\cdot$ & $\cdot$ & $\cdot$ & $\bullet$ & $\bullet$ & $\cdot$ & $\bullet$ & $\bullet$ & $\bullet$ & $\bullet$ & $\bullet$ & $\cdot$ & $\bullet$ & $\cdot$ & $\bullet$ & $\cdot$ & $\otimes$ & & & & & & \\
\hline 31. & Luna 7 & Luna E-6.No.11 & $\bullet$ & $\bullet$ & $\bullet$ & $\bullet$ & $\bullet$ & $\bullet$ & $\bullet$ & $\bullet$ & $\bullet$ & $\bullet$ & - & $\bullet$ & $\bullet$ & $\bullet$ & $\bullet$ & $\bullet$ & $\bullet$ & $\bullet$ & $\bullet$ & $\otimes$ & & & & & & \\
\hline 32. & Luna 8 & Luna E-6.No.12 & $\bullet$ & - & - & - & $\cdot$ & - & $\bullet$ & - & - & - & - & $\bullet$ & - & $\cdot$ & - & $\bullet$ & - & - & - & $\bullet$ & $\cdot$ & $\otimes$ & & & & \\
\hline 33. & Kosmos 111 & $\begin{array}{l}\text { Luna E-6S.No.204 } \\
\end{array}$ & $\bullet$ & $\bullet$ & $\bullet$ & $\bullet$ & $\bullet$ & $\otimes$ & & & & & & & & & & & & & & & & & & & & \\
\hline 34. & Luna 1966A & & $\otimes$ & & & & & & & & & & & & & & & & & & & & & & & & & \\
\hline 35. & Explorer 33 & AIMP-D & $\bullet$ & $\cdot$ & - & $\bullet$ & $\bullet$ & $\bullet$ & - & $\bullet$ & $\cdot$ & $\bullet$ & $\bullet$ & $\bullet$ & $\bullet$ & $\otimes$ & & & & & & & & & & & & \\
\hline
\end{tabular}




\begin{tabular}{|c|c|c|c|c|c|c|c|c|c|c|c|c|c|c|c|c|c|c|c|c|c|c|c|c|c|c|c|c|}
\hline S.No & Spacecraft & Distinct Name & LNH & BSB & ss & CRC-1 & EPOI & vss & OMB & CRC-2 & TLI & MCCM & LA & CRC-3 & OIB & LOI & LMS & CRC-4 & EITOI & TOI & ssc & DEI & CRC-5 & LD & LL & CRC-6 & LGTD & \begin{tabular}{|l|} 
CRC-7 \\
\end{tabular} \\
\hline 36. & Lunar Orbiter 1 & \begin{tabular}{|l|} 
Luna Orbiter-A \\
\end{tabular} & $\bullet$ & $\bullet$ & $\bullet$ & $\bullet$ & $\bullet$ & $\bullet$ & $\bullet$ & $\bullet$ & - & $\bullet$ & $\bullet$ & $\bullet$ & $\bullet$ & $\bullet$ & $\bullet$ & $\bullet$ & - & $\otimes$ & & & & & & & & \\
\hline 37. & Luna 11 & \begin{tabular}{|l|} 
E-6LF.No.101 \\
\end{tabular} & $\bullet$ & $\bullet$ & $\bullet$ & $\bullet$ & $\bullet$ & $\bullet$ & $\bullet$ & $\bullet$ & $\bullet$ & $\bullet$ & $\bullet$ & $\bullet$ & $\bullet$ & $\bullet$ & $\bullet$ & $\bullet$ & $\bullet$ & $\bullet$ & $\otimes$ & & & & & & & \\
\hline 38. & Surveyor 2 & \begin{tabular}{|l|} 
Surveyor-B \\
\end{tabular} & $\bullet$ & $\bullet$ & $\bullet$ & $\bullet$ & $\bullet$ & $\bullet$ & $\bullet$ & $\bullet$ & - & $\otimes$ & & & & & & & & & & & & & & & & \\
\hline 39. & Kosmos 154 & 7K-L1.No.3P & $\bullet$ & $\bullet$ & $\bullet$ & $\bullet$ & $\bullet$ & $\otimes$ & & & & & & & & & & & & & & & & & & & & \\
\hline 40. & Surveyor 4 & Surveyor-D & $\bullet$ & $\cdot$ & $\bullet$ & $\bullet$ & $\bullet$ & $\bullet$ & $\bullet$ & $\cdot$ & $\bullet$ & $\bullet$ & $\bullet$ & $\bullet$ & $\bullet$ & $\bullet$ & $\bullet$ & $\bullet$ & - & $\bullet$ & $\bullet$ & $\bullet$ & $\bullet$ & $\bullet$ & $\bullet$ & $\otimes$ & & \\
\hline 41. & Zond 1967A & \begin{tabular}{|l|} 
Soyuz 7K-L1.No.4L \\
\end{tabular} & $\bullet$ & $\otimes$ & & & & & & & & & & & & & & & & & & & & & & & & \\
\hline 42. & Zond 1967B & Soyuz 7L-L1.No.5L & $\bullet$ & $\otimes$ & & & & & & & & & & & & & & & & & & & & & & & & \\
\hline 43. & Luna $1968 \mathrm{~A}$ & Luna E-6LS.No.112 & $\bullet$ & $\otimes$ & & & & & & & & & & & & & & & & & & & & & & & & \\
\hline 44. & Zond 4 & 7K-L1.No.6L & $\bullet$ & $\bullet$ & $\bullet$ & $\bullet$ & $\bullet$ & $\bullet$ & - & $\bullet$ & - & $\bullet$ & $\bullet$ & $\bullet$ & $\bullet$ & $\bullet$ & $\bullet$ & $\bullet$ & $\bullet$ & $\bullet$ & $\bullet$ & $\bullet$ & - & $\otimes$ & & & & \\
\hline 45. & Zond 1968A & \begin{tabular}{|l|} 
Soyuz 7K-L1.7L \\
\end{tabular} & $\bullet$ & $\otimes$ & & & & & & & & & & & & & & & & & & & & & & & & \\
\hline 46. & Zond 6 & 7K-L1.No.12L & $\bullet$ & $\cdot$ & $\bullet$ & $\cdot$ & - & $\otimes$ & & & & & & & & & & & & & & & & & & & & \\
\hline 47. & Zond 1969A & \begin{tabular}{|l|} 
Soyuz 7K- \\
L1.No.13L \\
\end{tabular} & $\bullet$ & $\otimes$ & & & & & & & & & & & & & & & & & & & & & & & & \\
\hline 48. & Luna 1969A & \begin{tabular}{|l|} 
Luna E-8.No.201 \\
\end{tabular} & $\bullet$ & $\otimes$ & & & & & & & & & & & & & & & & & & & & & & & & \\
\hline 49. & Zond L1S-1 & \begin{tabular}{|l|} 
Soyuz 7K- \\
L1S.No.3S
\end{tabular} & $\otimes$ & & & & & & & & & & & & & & & & & & & & & & & & & \\
\hline 50. & Luna 1969B & & $\otimes$ & & & & & & & & & & & & & & & & & & & & & & & & & \\
\hline 51. & Luna 1969C & Luna E-8-5.No.402 & $\otimes$ & & & & & & & & & & & & & & & & & & & & & & & & & \\
\hline 52. & Zond L1S-2 & \begin{tabular}{|l|} 
Soyuz 7L- \\
L1S.No.5L \\
\end{tabular} & $\otimes$ & & & & & & & & & & & & & & & & & & & & & & & & & \\
\hline 53. & Luna 15 & E-8-5.No.401 & - & $\cdot$ & $\bullet$ & $\bullet$ & $\otimes$ & & & & & & & & & & & & & & & & & & & & & \\
\hline 54. & Kosmos 300 & E-8-5.No.403 & $\bullet$ & $\bullet$ & $\bullet$ & $\bullet$ & $\bullet$ & $\bullet$ & $\otimes$ & & & & & & & & & & & & & & & & & & & \\
\hline 55. & Kosmos 305 & E-8-5.No.404 & $\bullet$ & $\bullet$ & $\bullet$ & $\bullet$ & $\bullet$ & $\bullet$ & $\otimes$ & & & & & & & & & & & & & & & & & & & \\
\hline 56. & Luna 1970A & \begin{tabular}{|l|l} 
Luna E-8-5.No.405 \\
\end{tabular} & $\bullet$ & $\otimes$ & & & & & & & & & & & & & & & & & & & & & & & & \\
\hline 57. & Luna 1970B & & $\otimes$ & & & & & & & & & & & & & & & & & & & & & & & & & \\
\hline 58. & Soyuz 7K-LOK & 7K-L1E.No.1 & $\bullet$ & $\otimes$ & & & & & & & & & & & & & & & & & & & & & & & & \\
\hline 59. & Luna 18 & E-8-5.No.407 & $\bullet$ & $\bullet$ & $\bullet$ & $\bullet$ & $\bullet$ & $\bullet$ & $\bullet$ & $\bullet$ & - & $\bullet$ & $\bullet$ & $\bullet$ & $\bullet$ & $\bullet$ & $\bullet$ & $\bullet$ & $\bullet$ & $\bullet$ & $\bullet$ & $\bullet$ & $\bullet$ & $\otimes$ & & & & \\
\hline 60. & Soyuz 7K-LOK & 7K-LOK.No.6A & $\otimes$ & & & & & & & & & & & & & & & & & & & & & & & & & \\
\hline 61. & Luna 23 & \begin{tabular}{|l|} 
E-8-5M.No.410 \\
\end{tabular} & $\bullet$ & $\bullet$ & $\bullet$ & $\bullet$ & $\bullet$ & $\bullet$ & $\bullet$ & $\bullet$ & $\bullet$ & $\bullet$ & $\bullet$ & $\bullet$ & $\bullet$ & $\bullet$ & $\bullet$ & $\bullet$ & $\bullet$ & $\bullet$ & $\bullet$ & $\bullet$ & $\bullet$ & $\bullet$ & $\otimes$ & $\otimes$ & $\otimes$ & \\
\hline 62. & Luna 1975A & Luna E-8-5.No.412 & $\bullet$ & $\otimes$ & & & & & & & & & & & & & & & & & & & & & & & & \\
\hline 63. & Hiten & MUSES-A & $\bullet$ & $\bullet$ & $\bullet$ & $\bullet$ & $\bullet$ & - & - & $\bullet$ & $\bullet$ & - & - & $\bullet$ & $\bullet$ & $\bullet$ & $\bullet$ & $\otimes$ & & & & & & & & & & \\
\hline 64. & Hagoromo & & $\bullet$ & $\bullet$ & $\bullet$ & $\bullet$ & $\bullet$ & $\bullet$ & $\bullet$ & $\bullet$ & $\bullet$ & $\bullet$ & $\bullet$ & $\bullet$ & $\bullet$ & $\bullet$ & $\bullet$ & $\otimes$ & & & & & & & & & & \\
\hline 65. & HGS-1 & Asiasat-3/PAS-22 & - & $\cdot$ & $\bullet$ & $\bullet$ & $\bullet$ & $\otimes$ & & & & & & & & & & & & & & & & & & & & \\
\hline 66. & Nozomi & Planet-B & $\bullet$ & $\bullet$ & $\bullet$ & $\bullet$ & $\bullet$ & $\bullet$ & $\bullet$ & $\otimes$ & & & & & & & & & & & & & & & & & & \\
\hline 67. & Chandrayaan 1 & & $\bullet$ & $\bullet$ & $\bullet$ & $\bullet$ & $\bullet$ & $\bullet$ & $\bullet$ & $\cdot$ & $\bullet$ & $\bullet$ & $\bullet$ & $\bullet$ & $\bullet$ & $\otimes$ & & & & & & & & & & & & \\
\hline 68. & Longjiang 1 & DSWLP-A1/LO-93 & $\bullet$ & $\bullet$ & $\bullet$ & $\bullet$ & $\bullet$ & $\bullet$ & $\bullet$ & $\bullet$ & $\bullet$ & $\bullet$ & $\bullet$ & $\bullet$ & $\bullet$ & $\otimes$ & & & & & & & & & & & & \\
\hline 69. & Beresheet & & $\bullet$ & $\bullet$ & $\bullet$ & $\bullet$ & $\bullet$ & $\bullet$ & $\bullet$ & $\bullet$ & $\bullet$ & $\bullet$ & $\bullet$ & $\bullet$ & $\bullet$ & $\bullet$ & $\bullet$ & $\bullet$ & $\bullet$ & $\bullet$ & $\bullet$ & $\bullet$ & $\bullet$ & $\otimes$ & $\otimes$ & & & \\
\hline 70. & Vikram/Pragyan & & $\bullet$ & $\bullet$ & $\bullet$ & $\bullet$ & $\bullet$ & $\bullet$ & - & $\cdot$ & - & $\bullet$ & $\bullet$ & $\bullet$ & $\bullet$ & $\bullet$ & $\bullet$ & $\bullet$ & $\bullet$ & - & $\bullet$ & $\bullet$ & $\cdot$ & $\otimes$ & $\otimes$ & & & \\
\hline
\end{tabular}




\section{Acknowledgments}

The main author Malaya Kumar Biswal M would like to dedicate this work to his beloved mother late. Mrs. Malathi Biswal for her motivational speech and emotional support throughout his life.

\section{References}

[1] McDowell, Jonathan C. (Nov 2019). "The Deep Space Catalog V1.0" Accessed from https://www.planet4589.org/space/deepcat/index.html on 20 March 2020.

[2] Moroz, V. I., Huntress, W. T., \& Shevalev, I. L. (2002). Planetary missions of the 20th century. Cosmic Research, 40(5), 419-445

[3] Huntress, W. T., Moroz, V. I., \& Shevalev, I. L. (2003). Lunar and planetary robotic exploration missions in the 20th century. Space science reviews, 107(3-4), 541-649.

[4] Siddiqi, A. A., \& Launius, R. (2002). Deep space chronicle: A chronology of deep space and planetary probes 1958-2000.

[5] Bleeker, J. A., Geiss, J., \& Huber, M. C. (2001). The century of space science. In The Century of Space Science (pp. 3-22). Springer, Dordrecht.

[6] Biswal M, Malaya Kumar; Annavarapu, Ramesh Naidu (2020), “Chronology of Mars Exploration Missions 1960-2020”, Mendeley Data, v1. http://dx.doi.org/10.17632/8nnmzsgztf.1

[7] Chronology of Mars Exploration. NASA. https://nssdc.gsfc.nasa.gov/planetary/chronology mars.html on 20 March 2020.

[8] Mission to Mars. Weebau Space Encyclopedia. Accessed from https://weebau.com/ on 20 March 2020.

[9] Chronology of Space Launches. Gunter's Space Page. Accessed from https://space.skyrocket.de/directories/chronology.htm on 20 March 2020.

[10] Mission to Mars. Weebau Space Encyclopedia. Accessed from https://weebau.com/ on 20 March 2020.

[11] Chronology of Space Launches. Gunter's Space Page. Accessed from https://space.skyrocket.de/directories/chronology.htm on 20 March 2020.

[12] Wikipedia contributors. (2020, April 18). List of missions to Mars. In Wikipedia, The Free Encyclopedia. Accessed from https://en.wikipedia.org/w/index.php?title=List_of_missions_to_Mars\&oldid=951759136 on 20 March 2020.

[13] Biswal M, Malaya Kumar \& Annavarapu, Ramesh Naidu. (2019). Chronology of Mars Exploration Missions. 10.13140/RG.2.2.36435.22560.

[14] Biswal M, M. K., \& Naidu A, R. (2019). Mars Missions Failure Report Assortment: Review and Conspectus. arXiv preprint arXiv:1903.02461.

[15] Wikipedia contributors. (2020, April 27). List of missions to the Moon. In Wikipedia, The Free Encyclopedia. Accessed from https://en.wikipedia.org/w/index.php?title=List_of_missions_to_the_Moon\&oldid=953538613 on 20 March 2020 .

[16] Biswal M, Malaya Kumar \& Annavarapu, Ramesh Naidu. (2020). Mars Missions Failure Report Assortment - Presentation Slides. 10.13140/RG.2.2.16250.85441. 